\title{
Drell-Yan hadron tensor: Contour gauge and gluon propagator
}

\author{
I. V. Anikin, ${ }^{1, *}$ I. O. Cherednikov, ${ }^{2,3, \dagger}$ and O. V. Teryaev ${ }^{1, \$}$ \\ ${ }^{1}$ Bogoliubov Laboratory of Theoretical Physics, JINR, 141980 Dubna, Russia \\ ${ }^{2}$ SCK-CEN, B-2400 Mol, Belgium \\ ${ }^{3}$ Departement Fysica, Universiteit Antwerpen, B-2020 Antwerpen, Belgium
}

(Received 14 December 2016; published 23 February 2017)

\begin{abstract}
We consider the gauge invariant Drell-Yan hadron tensor which includes the standard and nonstandard diagram contributions. The nonstandard diagram contribution appeared owing to the complexity of the twist three $B^{V}\left(x_{1}, x_{2}\right)$-function where the gluon pole manifests. We use the contour gauge conception which allows us to fix easily the spurious uncertainties in the gluon propagator. The contour gauge condition is generated by the corresponding Wilson lines in both the standard and nonstandard diagrams. We demonstrate the substantial role of the nonstandard diagram for forming of the relevant contour in the Wilson path-ordered exponential that leads to the spurious singularity fixing.
\end{abstract}

DOI: 10.1103/PhysRevD.95.034032

\section{INTRODUCTION}

The investigation of the nucleon (hadron) composite structure is still the most important subject of hadron physics. From the experimental point of view, one of the widespread and useful instruments for such studies is the single spin asymmetry (SSA). Especially, the single transverse spin asymmetry opens access to the threedimensional nucleon structure thanks to the nontrivial connection between the transverse spin and the parton transverse momentum dependence (see, for example, [1-5]).

In QCD, the SSA related to the Drell-Yan (DY) process was first considered in the case of the longitudinally polarized hadron [6,7]. This SSA is especially interesting provided the second hadron is a pion. This is because of the sensitivity $[8,9]$ to the shape of pion distribution amplitude, being currently the object of major interest $[10,11]$ (see also [12] and the references therein). It was shown that the imaginary phase in the SSA which is associated with the longitudinally polarized nucleon appears due to either the hard perturbative gluon loops [6,7] or twist four contribution of the pion distribution amplitude $[8,9]$.

For the single transverse spin asymmetry in the transverse-polarized DY process, the imaginary part has previously been extracted from the quark propagator in the so-called standard [see Fig. 1 (left panel)] diagram with quark-gluon twist three correlator only (it leads to the gluon pole contribution to SSA, see $[13,14])$. The reason was that the ambiguity in the boundary conditions provides the purely real quark-gluon function $B^{V}\left(x_{1}, x_{2}\right)$ which parametrizes the $\left\langle\bar{\psi} \gamma^{+} A^{\perp} \psi\right\rangle$ matrix element. On the other hand, the real $B^{V}\left(x_{1}, x_{2}\right)$-function kills the contribution from the nonstandard [see Fig. 1 (right panel)] diagram which

\footnotetext{
*anikin@theor.jinr.ru

igor.cherednikov@uantwerpen.be

*teryaev@theor.jinr.ru
}

however is absolutely necessary to ensure the QED gauge invariance of the DY hadron tensor. This situation has been discussed in detail in a series of papers [15] where, with the help of the contour gauge conception, the twist three $B^{V}\left(x_{1}, x_{2}\right)$-function has been proven to be in fact the complex function. In turn, this leads to the nonzero contribution from the nonstandard diagram which produces the imaginary phase required to have the SSA. This additional contribution also leads to an extra factor of 2 for SSA.

Recently, the problem of the spurious singularity fixing in the (local) axial gauge has attracted attention again (see, for example, $[3,16,17])$.

The light-cone axial gauge condition imposed on the non-Abelian gluon field, $A^{+}=0$, naturally enables the parton number (probability) interpretation of parton density functions in the tree level $[18,19]$. However, perturbative calculations beyond the tree approximation demand careful treatment of the so-called spurious uncertainties in the gluon propagator $D_{\mu \nu}(k)$ in the light-cone gauge [20-23]. The latter arise as ill-defined pole singularities of the form $\sim\left(k^{+}\right)^{-1}$ and are associated, putting the same issue a bit different, with the residual gauge freedom due to incomplete gauge fixing by $A^{+}=0$. For this reason calculations in the axial (light-cone) gauge in higher perturbative orders are cumbersome and sometimes even contradictory $[24,25]$. One can attempt to overcome this difficulty by working in the well-defined general covariant gauge setting the gauge parameter to $\xi=-3+0\left(\alpha_{s}\right)$, which is known to effectively "imitate" noncovariant gauges [25,26]. Another approach is to keep working in the light-cone gauge and to get rid of the residual gauge freedom by an appropriate extra gauge-fixing condition. The latter can be obtained in terms of the various boundary conditions for the gluon fields and/or their spatial derivatives $[3,16,17]$.

In the present work, we investigate an alternative approach to formulation of the more general gauge-fixing 

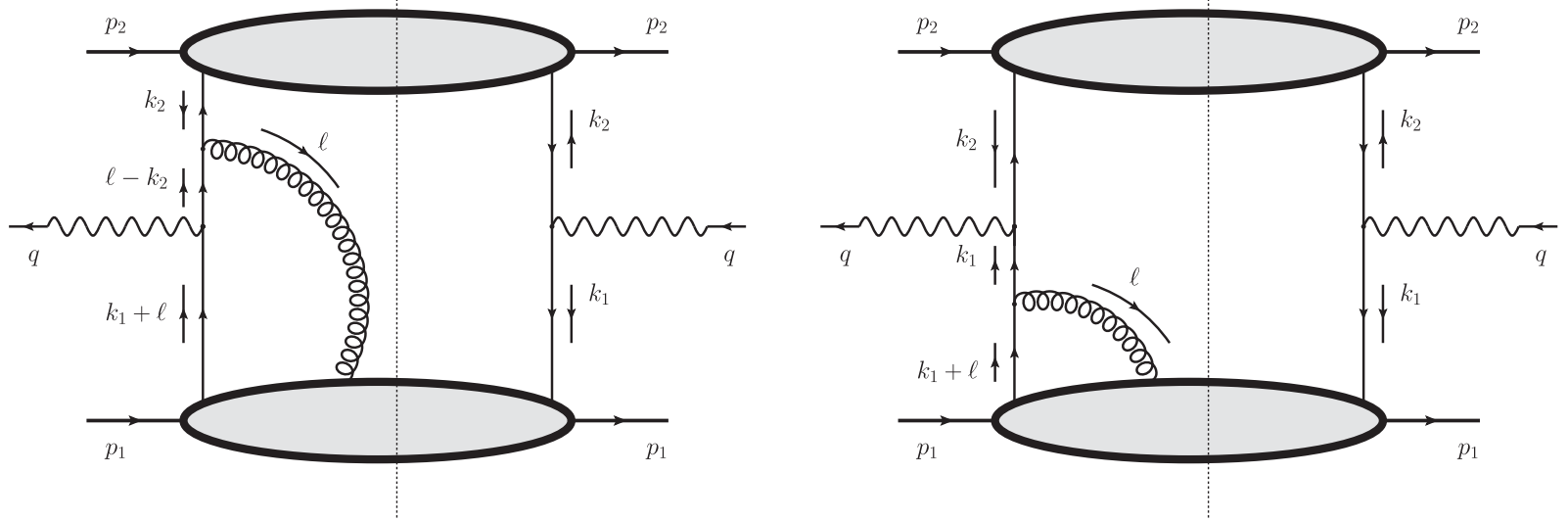

FIG. 1. The Feynman diagrams which contribute to the polarized Drell-Yan hadron tensor: the standard (left panel) and nonstandard diagrams (right panel).

condition from the very beginning which is supposed to entail the "right" pole prescriptions for the gluon propagator. We continue to explore the contour gauge conception and demonstrate how the spurious uncertainties in the gluon propagator can ultimately be fixed in the nonlocal axial gauges. Working within the collinear factorization procedure, we emphasize the substantial role of the nonstandard diagram to get the relevant contour in the Wilson path-ordered exponential needed to fix ultimately the spurious singularity in the gluon propagator.

\section{GETTING STARTED: KINEMATICS}

We begin with the kinematics of Drell-Yan process. As in [15], we study the Drell-Yan process with the transversely polarized hadron:

$$
\begin{aligned}
& N^{(\uparrow \downarrow)}\left(p_{1}\right)+N\left(p_{2}\right) \rightarrow \gamma^{*}(q)+X\left(P_{X}\right) \\
& \quad \rightarrow \ell\left(l_{1}\right)+\bar{\ell}\left(l_{2}\right)+X\left(P_{X}\right),
\end{aligned}
$$

where the virtual photon producing the lepton pair $\left(l_{1}+l_{2}=q\right)$ has a large mass squared $\left(q^{2}=Q^{2}\right)$ while the transverse momenta are small and integrated out. This kinematics (anticipating the collinear factorization procedure) suggests a convenient frame with fixed dominant light-cone directions [15]:

$$
\begin{aligned}
p_{1} & \approx \frac{Q}{x_{B} \sqrt{2}} n^{*}, \quad p_{2} \approx \frac{Q}{y_{B} \sqrt{2}} n, \\
n^{* \mu} & =\left(\frac{1}{\sqrt{2}}, \overrightarrow{\mathbf{0}}_{\perp}, \frac{1}{\sqrt{2}}\right)=\left(1^{+}, 0^{-}, \overrightarrow{\mathbf{0}}_{\perp}\right), \\
n^{\mu} & =\left(\frac{1}{\sqrt{2}}, \overrightarrow{\mathbf{0}}_{\perp}, \frac{-1}{\sqrt{2}}\right)=\left(0^{+}, 1^{-}, \overrightarrow{\mathbf{0}}_{\perp}\right), \\
n^{*} \cdot n & =n^{*+} n^{-}=1 .
\end{aligned}
$$

It is also instructive to introduce the dimensionful analogs of $n, n^{*}$ as

$$
\tilde{n}^{-}=\frac{p_{2}^{-}}{p_{1} p_{2}}, \quad \breve{n}^{+}=\frac{p_{1}^{+}}{p_{1} p_{2}} .
$$

With the above vectors as a basis, an arbitrary vector can be (Sudakov) decomposed as

$$
\begin{gathered}
a^{\mu}=a^{+} n^{* \mu}+a^{-} n^{\mu}+a_{\perp}^{\mu}, \\
a^{\mu,+} \stackrel{\text { def }}{=} a^{+} n^{* \mu}, \quad a^{\mu,-} \stackrel{\text { def }}{=} a^{-} n^{\mu} .
\end{gathered}
$$

In what follows we will not be so precise about writing the covariant and contravariant vectors in any kinds of summations over the four-dimensional vectors, except the cases where this trick may lead to misunderstanding.

\section{DRELL-YAN HADRON TENSOR: DERIVATION OF WILSON LINES}

The polarized DY process is a very convenient process to study the role of twist three by exploring different kinds of SSAs. For example, one can study the left-right asymmetry which means the transverse momenta of the leptons are correlated with the direction $\mathbf{S} \times \mathbf{e}_{z}$ where $S_{\mu}$ implies the transverse polarization vector of the nucleon and $\mathbf{e}_{z}$ is a beam direction [27].

Generally speaking, any single spin asymmetries can be presented in the symbolical form as

$$
\mathcal{A} \sim d \sigma^{(\uparrow)}-d \sigma^{(\downarrow)} \sim \mathcal{L}_{\mu \nu} \mathcal{W}_{\mu \nu},
$$

where $\mathcal{L}_{\mu \nu}$ is an unpolarized leptonic tensor and $\mathcal{W}_{\mu \nu}$ stands for the hadronic tensor. At the moment, we do not specify the phase space in Eq. (5) because the exact expression for SSA is irrelevant for our discussion. Instead, we mainly pay attention to the hadron tensor which can be presented as

$$
\begin{aligned}
\mathcal{W}_{\mu \nu}= & \mathcal{W}_{\mu \nu}^{(0)}+\mathcal{W}_{\mu \nu}^{(1)}(g \mid A)+\mathcal{W}_{\mu \nu}^{(2)}(g \mid A) \\
& +\left(g^{n} \text {-terms } \mid n \geq 2\right) \\
= & \overline{\mathcal{W}}_{\mu \nu}^{(0)}\left(A^{ \pm}\right)+\mathcal{W}_{\mu \nu}^{(1)}\left(g \mid A^{\perp}\right)+\mathcal{W}_{\mu \nu}^{(2)}\left(g \mid A^{\perp}\right)+\cdots,
\end{aligned}
$$


where $g$ denotes the strong interaction coupling constant and

$\overline{\mathcal{W}}_{\mu \nu}^{(0)}\left(A^{ \pm}\right)=\mathcal{W}_{\mu \nu}^{(0)}+\mathcal{W}_{\mu \nu}^{(1)}\left(g \mid A^{+}\right)+\mathcal{W}_{\mu \nu}^{(2)}\left(g \mid A^{-}\right)+\cdots$.

The hadron tensor representations can be found below. In our case, the single transverse spin asymmetry is only generated by the hadron tensors $\mathcal{W}_{\mu \nu}^{(1)}\left(g \mid A^{\perp}\right)$ and $\mathcal{W}_{\mu \nu}^{(2)}\left(g \mid A^{\perp}\right)$ where the twist three contributions related to $\left\langle\bar{\psi} \gamma^{+} A^{\perp} \psi\right\rangle$ have been extracted. As shown below, the $\left\langle\bar{\psi} \gamma^{+} A^{ \pm} \psi\right\rangle$-correlators in the hadron tensors $\mathcal{W}_{\mu \nu}^{(1,2)}(g \mid A)$ participate in forming the corresponding Wilson lines which appear in the quark-antiquark correlators of the hadron tensor $\overline{\mathcal{W}}_{\mu \nu}^{(0)}\left(A^{ \pm}\right)$. In the frame of usual axial gauge $\left(A^{+}=0\right)$, this kind of contribution can be discarded. However, we work in the contour gauge which is, first, a nonlocal generalization of the well-known axial gauge. Second, the contour gauge contains the important and unique additional information (needed to fix the prescription in the gluon poles) which is invisible in the case of usual (local) axial gauge. From this point of view, before we discard the terms with $A^{+}$, we have to determine the relevant fixed path in the restored Wilson line with $A^{+}$ which eventually leads to certain prescriptions in the gluon poles (for further explanations, see [15]).

\section{A. The standard hadron tensor (direct process)}

In this section, we analyze the part of the DY hadron tensor which is generated by the diagram in Fig. 1 (left panel). This is the standard hadron tensor which can be written in nonfactorized form as

$$
\begin{aligned}
& \mathcal{W}_{\mu \nu}^{(1)}(g \mid A) \\
& =\int d^{4} k_{1} d^{4} k_{2} \delta^{(4)}\left(k_{1}+k_{2}-q\right) \bar{\Phi}^{\left[\gamma^{-}\right]}\left(k_{2}\right) \\
& \quad \times \int d^{4} \ell \Phi_{\alpha}^{(A)\left[\gamma^{+}\right]}\left(k_{1}, \ell\right) \operatorname{tr}\left[\gamma_{\mu} \gamma^{-} \gamma_{\nu} \gamma^{+} \gamma_{\alpha}\right. \\
& \quad \times \frac{\left.\left(\ell^{+}-k_{2}^{+}\right) \gamma^{-}+\left(\ell^{-}-k_{2}^{-}\right) \gamma^{+}-\left(\vec{\ell}_{\perp}-\vec{k}_{2 \perp}\right) \vec{\gamma}_{\perp}\right]}{\left(\ell-k_{2}\right)^{2}+i \varepsilon},
\end{aligned}
$$

where

$$
\begin{gathered}
\Phi_{\alpha}^{(A)\left[\gamma^{+}\right]}\left(k_{1}, \ell\right) \stackrel{\mathcal{F}_{2}}{=}\left\langle p_{1}, S^{T}\left|\bar{\psi}\left(\eta_{1}\right) \gamma^{+} g A_{\alpha}(z) \psi(0)\right| S^{T}, p_{1}\right\rangle, \\
\bar{\Phi}^{\left[\gamma^{-}\right]}\left(k_{2}\right) \stackrel{\mathcal{F}_{1}}{=}\left\langle p_{2}\left|\bar{\psi}\left(\eta_{2}\right) \gamma^{-} \psi(0)\right| p_{2}\right\rangle .
\end{gathered}
$$

In Eqs. (9) and (10), $\mathcal{F}_{1}$ and $\mathcal{F}_{2}$ denote the Fourier transformation with the measures defined as

$$
d^{4} \eta_{2} e^{i k_{2} \cdot \eta_{2}} \quad \text { and } \quad d^{4} \eta_{1} d^{4} z e^{-i k_{1} \cdot \eta_{1}-i \ell \cdot z}
$$

respectively. For the sake of shortness, we will omit $S^{T}$ in the hadron states which indicates the transverse polarization of hadron.

We now analyze the tensor structure of the trace in Eq. (8). We can see that the first term of the quark propagator, $\left.\ell^{+}-k_{2}^{+}\right) \gamma^{-}$, singles out only the transverse components of gluon field in the quark-gluon correlator, see Eq. (9). At the same time, the second term of the quark propagator, $\left(\ell^{-}-k_{2}^{-}\right) \gamma^{+}$, separates out only the longitudinal component $A^{+}$in the quark-gluon correlator. This second term is very important for derivation of the corresponding Wilson line which defines in our approach the contour gauge. The third term of the quark propagator gives us the quark-gluon correlator with both indices $\alpha=(+, \perp)$.

The collinear factorization procedure for the process under consideration can be introduced by the following steps (for details see, e.g., Refs. [28,29]):

(a) the decomposition of loop integration momenta around the corresponding dominant direction:

$$
k_{i}=x_{i} p+\left(k_{i} \cdot p\right) n+k_{T}
$$

within the certain light cone basis formed by the vectors $p$ and $n$ (in our case, $n^{*}$ and $n$ );

(b) the replacement:

$$
d^{4} k_{i} \Rightarrow d^{4} k_{i} d x_{i} \delta\left(x_{i}-k_{i} \cdot n\right)
$$

that introduces the fractions with the appropriated spectral properties;

(c) the decomposition of the corresponding propagator products, which will finally form the hard part, around the dominant direction. It is necessary to notice that in the DY process case the corresponding $\delta$-functions which appeared in the hadron tensor and expressed the momentum conservation law should be also referred to the hard parts. This statement was argued in [30] in the context of the so-called factorization links;

(d) the use of the collinear Ward identity if it is necessary within the given accuracy level;

(e) performing the Fierz decomposition for $\psi_{\alpha}(z) \bar{\psi}_{\beta}(0)$ in the corresponding space up to the needed projections.

Let us first dwell on the second term, $\left(\ell^{-}-k_{2}^{-}\right) \gamma^{+}$, contribution. This term is responsible for forming the Wilson line in the gauge-invariant quark-antiquark string operator. Indeed, making used the collinear factorization $\left(\ell^{-} \approx 0,\left(\ell-k_{2}\right)^{2} \approx-2 \ell^{+} k_{2}^{-}\right)$, the above-mentioned term contributes in the hadron tensor as

$$
\begin{aligned}
\mathcal{W}_{\mu \nu}^{(1)\left[k_{2}^{-}\right]}\left(g \mid A^{+}\right)= & \int d \mu\left(k_{i} ; x_{1}, y\right) \bar{\Phi}^{\left[\gamma^{-}\right]}\left(k_{2}\right) \frac{1}{2} \\
& \times \int d z^{-} \operatorname{tr}\left[\gamma_{\mu} \gamma^{-} \gamma_{\nu} \gamma^{+} \gamma^{-} \gamma^{+}\right] \\
& \times \int d \ell^{+} \frac{e^{-i \ell^{+} z^{-}}}{\ell^{+}-i \varepsilon} \int d^{4} \eta_{1} e^{-i k_{1} \cdot \eta_{1}} \\
& \times\left\langle p_{1}\left|\bar{\psi}\left(\eta_{1}\right) \gamma^{+} g A^{+}\left(0^{+}, z^{-}, \overrightarrow{\mathbf{0}}_{\perp}\right) \psi(0)\right| p_{1}\right\rangle,
\end{aligned}
$$


where the integration measure reads

$$
\begin{aligned}
d \mu\left(k_{i} ; x_{1}, y\right)= & d x_{1} d^{4} k_{1} \delta\left(x_{1}-\frac{k_{1}^{+}}{p_{1}^{+}}\right) d y d^{4} k_{2} \delta\left(y-\frac{k_{2}^{-}}{p_{2}^{-}}\right) \\
& \times\left[\delta^{(4)}\left(x_{1} p_{1}+y p_{2}-q\right)\right] .
\end{aligned}
$$

The prescription $-i \varepsilon$ in the denominator of (12) directly follows from the standard causal prescription for the massless quark propagator in (8) (cf. [31]).

Integration over $\ell^{+}$in (12), using the well-known integral representation

$$
\theta( \pm x)=\frac{ \pm i}{2 \pi} \int_{-\infty}^{+\infty} d k \frac{e^{-i k x}}{k \pm i \varepsilon}
$$

leads to the following expression:

$$
\begin{aligned}
& \mathcal{W}_{\mu \nu}^{(1)\left[k_{2}^{-}\right]}\left(g \mid A^{+}\right) \\
& =\int d \mu\left(k_{i} ; x_{1}, y\right) \operatorname{tr}\left[\gamma_{\mu} \gamma^{-} \gamma_{\nu} \gamma^{+}\right] \bar{\Phi}^{\left[\gamma^{-}\right]}\left(k_{2}\right) \int d^{4} \eta_{1} e^{-i k_{1} \cdot \eta_{1}} \\
& \quad \times\left\langle p_{1}\left|\bar{\psi}\left(\eta_{1}\right) \gamma^{+} i g \int_{-\infty^{-}}^{0^{-}} d z^{-} A^{+}\left(0^{+}, z^{-}, \overrightarrow{\mathbf{0}}_{\perp}\right) \psi(0)\right| p_{1}\right\rangle,
\end{aligned}
$$

where we use

$$
\frac{1}{2} \gamma^{+} \gamma^{-} \gamma^{+}=\gamma^{+}
$$

It is important to stress that the leading order hadron tensor $\mathcal{W}_{\mu \nu}^{(0)}\left(g^{0}\right)$ differs from the hadron tensor (8) by overall sign: the leading hadron tensor has a prefactor $i^{2}$ due to two photon vertices, while the hadron tensor (8) is accompanied by a prefactor $i^{4}$ thanks to two photon and one gluon vertices together with the prefactor from the massless quark propagator $(-1) / i$ (we use the convention as in [32]).

Thus, if we include all gluon emissions from the antiquark going from the upper blob in Fig. 1 (left panel) (the so-called initial state interactions), we are able to get the corresponding $P$-exponential in $\Phi_{\alpha}^{(A)\left[\gamma^{+}\right]}\left(k_{1}, \ell\right)$. The latter is now represented by the following matrix element:

$\int d^{4} \eta_{1} e^{-i k_{1} \cdot \eta_{1}}\left\langle p_{1}\left|\bar{\psi}\left(\eta_{1}\right) \gamma^{+}\left[-\infty^{-} ; 0^{-}\right]_{A^{+}} \psi(0)\right| p_{1}\right\rangle$,

where

$$
\begin{aligned}
{\left[-\infty^{-} ; 0^{-}\right]_{A^{+}} } & \equiv\left[0^{+},-\infty^{-}, \overrightarrow{\boldsymbol{0}}_{\perp} ; 0^{+}, 0^{-}, \overrightarrow{\mathbf{0}}_{\perp}\right]_{A^{+}} \\
& =\mathbb{P} \exp \left\{i g \int_{0^{-}}^{-\infty^{-}} d z^{-} A^{+}\left(0^{+}, z^{-}, \overrightarrow{\mathbf{0}}_{\perp}\right)\right\}
\end{aligned}
$$

The collinear twist $\left(t=d-s_{a}\right)$ of $A^{+}$is equal to zero, therefore the Wilson line which is summing up all these components does not affect the twist expansion within the collinear factorization.

If now we include in our consideration the gluon emission from the incoming antiquark (the mirror contributions), we will obtain the Wilson line $\left[\eta_{1}^{-},-\infty^{-}\right]$which will ultimately give us, together with (18), the Wilson line connecting the points 0 and $\eta_{1}$ in (17) contributing to $\overline{\mathcal{W}}_{\mu \nu}^{(0)}$. This is exactly what happens, say, in the spin-averaged DY process [33]. However, for the SSA, these two diagrams should be considered individually. Indeed, their contributions to SSAs, contrary to the spin-averaged case, differ in sign and the dependence on the boundary point at $-\infty^{-}$ does not cancel.

For the pedagogical reason, we want to show the exponentiation of the transverse gluon field (here, we mainly follow to [16]), although we are restricted by the twist three case and the inclusion of all degrees of the transverse gluon field exceeds our accuracy. Let us consider the third term, $\left(\vec{\ell}_{\perp}-\vec{k}_{2 \perp}\right) \vec{\gamma}_{\perp}$, contribution which helps us to demonstrate the exponentiation of the transverse gluon fields. The corresponding hadron tensor part takes the following form:

$$
\begin{aligned}
& \mathcal{W}_{\mu \nu}^{(1)\left[\vec{e}_{\perp}\right]}\left(g \mid A^{\perp}\right) \\
& =\int d \mu\left(k_{i} ; x_{1}, y\right) \bar{\Phi}^{\left[\gamma^{-}\right]}\left(k_{2}\right) \operatorname{tr}\left[\gamma_{\mu} \gamma^{-} \gamma_{\nu} \gamma^{+} \gamma_{\alpha}^{\perp} \vec{\gamma}_{i}^{\perp}\right] \\
& \quad \times \int d^{4} \ell \frac{\left(\vec{\ell}^{\perp}-\vec{k}_{2}^{\perp}\right)_{i}}{2 \ell^{+} k_{2}^{-}+\vec{\ell}_{\perp}^{2}-i \varepsilon} \Phi_{\alpha}^{\left(A^{\perp}\right)\left[\gamma^{+}\right]}\left(k_{1}, \ell\right) \\
& \equiv \int d \mu\left(k_{i} ; x_{1}, y\right) \bar{\Phi}^{\left[\gamma^{-}\right]}\left(k_{2}\right) \operatorname{tr}\left[\gamma_{\mu} \gamma^{-} \gamma_{\nu} \gamma^{+} \gamma_{\alpha}^{\perp} \vec{\gamma}_{i}^{\perp}\right] \mathfrak{Q}_{i, \alpha},
\end{aligned}
$$

where we assume that $\vec{k}_{2 \perp} \approx 0$. In Eq. (15) let us focus on the $\ell$-integration, we have

$$
\begin{aligned}
\mathfrak{Q}_{i, \alpha}= & \int d \ell^{+} d \ell^{-} d^{2} \vec{\ell}_{\perp} \frac{\vec{\ell}_{i}^{\perp}}{2 \ell^{+} k_{2}^{-}+\vec{\ell}_{\perp}^{2}-i \varepsilon} \\
& \times \int d^{4} \eta_{1} d^{4} z e^{-i k_{1} \eta_{1}-i \ell z}\left\langle p_{1}\left|\bar{\psi}\left(\eta_{1}\right) \gamma^{+} g A_{\alpha}^{\perp}(z) \psi(0)\right| p_{1}\right\rangle .
\end{aligned}
$$

We now use the $\alpha$-representation for the denominator that stems from the quark propagator:

$$
\frac{1}{2 \ell^{+} k_{2}^{-}+\vec{\ell}_{\perp}^{2}-i \varepsilon}=i \int_{0}^{\infty} d \alpha e^{-i \alpha\left[2 \ell^{+} k_{2}^{-}+\vec{\ell}_{\perp}^{2}-i \varepsilon\right]} .
$$

Next, in Eq. (20) we perform the integrations over $d \ell^{-}$ and $d \ell^{+}$which give $\delta\left(z^{+}\right)$and $\delta\left(z^{-}+2 \alpha k_{2}^{-}\right)$, respectively. We remind that the variables $\alpha$ in (21) are dimensionful and $\operatorname{dim}_{M}[\alpha]=-2$. 
Therefore, the integral $\mathfrak{\&}$ takes the following form (cf. [16]):

$$
\begin{aligned}
\mathfrak{Q}_{i, \alpha}= & i \int d^{2} \vec{\ell}_{\perp} \vec{\ell}_{i}^{\perp} \int_{0}^{\infty} d \alpha e^{-i \alpha\left[\vec{\ell}_{\perp}^{2}-i \varepsilon\right]} \\
& \times \int d^{4} \eta_{1} d^{2} \vec{z}_{\perp} e^{-i k_{1} \eta_{1}+i \vec{\ell}_{\perp} \vec{z}_{\perp}} \\
& \times\left\langle p_{1}\left|\bar{\psi}\left(\eta_{1}\right) \gamma^{+} g A_{\alpha}^{\perp}\left(0^{+},-\infty^{-}, \vec{z}_{\perp}\right) \psi(0)\right| p_{1}\right\rangle .
\end{aligned}
$$

In Eq. (22) the transverse gluon field operator can be presented as

$A_{\alpha}^{\perp}\left(0^{+},-\infty^{-}, \vec{z}_{\perp}\right)=\frac{\partial}{\partial z_{\alpha}^{\perp}} \int_{\mathbb{C}}^{z^{\perp}} d \omega_{\beta}^{\perp} A_{\beta}^{\perp}\left(0^{+},-\infty^{-}, \vec{\omega}^{\perp}\right)$,

where we fix the arbitrary constant $\mathbb{C}$ to be $-\vec{\infty}_{\perp}$. By making use of the representation (23), after integration over $\alpha$ we arrive at

$$
\begin{aligned}
\mathfrak{Q}_{i, \alpha}= & i \int d^{2} \vec{\ell}_{\perp} \frac{\vec{\ell}_{i}^{\perp} \ell_{\alpha}^{\perp}}{\vec{\ell}_{\perp}^{2}-i \varepsilon} \int d^{4} \eta_{1} d^{2} \vec{z}_{\perp} e^{-i k_{1} \eta_{1}+i \vec{\ell}_{\perp} \vec{z}_{\perp}} \\
& \times\left\langle p_{1}\left|\bar{\psi}\left(\eta_{1}\right) \gamma^{+} g \int_{-\infty^{\perp}}^{z^{\perp}} d \omega_{\beta}^{\perp} A_{\beta}^{\perp}\left(0^{+},-\infty^{-}, \vec{\omega}^{\perp}\right) \psi(0)\right| p_{1}\right\rangle .
\end{aligned}
$$

We insert the obtained expression for $\mathfrak{Q}_{i, \alpha}$, see Eq. (24), into the expression for hadron tensor (15). After integration over $d^{2} \vec{\ell}_{\perp}$ and, then, after integration over $d^{2} \vec{z}_{\perp}$ we get the following expression for the $\vec{\ell}_{\perp}$-term of the hadron tensor:

$$
\begin{aligned}
& \mathcal{W}_{\mu \nu}^{(1)\left[\vec{e}_{\perp}\right]}\left(g \mid A^{\perp}\right) \\
& =\int d \mu\left(k_{i} ; x_{1}, y\right) \bar{\Phi}^{\left[\gamma^{-}\right]}\left(k_{2}\right) \operatorname{tr}\left[\gamma_{\mu} \gamma^{-} \gamma_{\nu} \gamma^{+}\right] \int d^{4} \eta_{1} e^{-i k_{1} \eta_{1}} \\
& \quad \times\left\langle p_{1}\left|\bar{\psi}\left(\eta_{1}\right) \gamma^{+} i g \int_{-\infty^{\perp}}^{0^{\perp}} d \omega_{\beta}^{\perp} A_{\beta}^{\perp}\left(0^{+},-\infty^{-}, \vec{\omega}^{\perp}\right) \psi(0)\right| p_{1}\right\rangle .
\end{aligned}
$$

As well as for the case of longitudinal gluons, if we now include all gluon emissions from the antiquark going from the upper blob in Fig. 1(left panel), we reproduce the corresponding $P$-exponential with the transverse gluons in $\Phi_{\alpha}^{(A)\left[\gamma^{+}\right]}\left(k_{1}, \ell\right)$. Together with the result obtained above for the $A^{+}$-fields, we finally have

$$
\begin{aligned}
\int d^{4} \eta_{1} e^{-i k_{1} \cdot \eta_{1}}\left\langle p_{1}\right| \bar{\psi}\left(0^{+}, \eta_{1}^{-}, \overrightarrow{\mathbf{0}}_{\perp}\right) \gamma^{+} \\
\quad \times\left[0^{+},-\infty^{-}, \overrightarrow{\mathbf{0}}_{\perp} ; 0^{+}, 0^{-}, \overrightarrow{\mathbf{0}}_{\perp}\right]_{A^{+}} \\
\quad \times\left[0^{+},-\infty^{-},-\vec{\infty}_{\perp} ; 0^{+},-\infty^{-}, \overrightarrow{\mathbf{0}}_{\perp}\right]_{A^{\perp}} \psi(0)\left|p_{1}\right\rangle,
\end{aligned}
$$

where

$$
\begin{aligned}
& {\left[0^{+},-\infty^{-},-\vec{\infty}_{\perp} ; 0^{+},-\infty^{-}, \overrightarrow{\mathbf{0}}_{\perp}\right]_{A^{\perp}}} \\
& \quad=\mathbb{P} \exp \left\{i g \int_{0^{\perp}}^{-\infty^{\perp}} d \omega_{\beta}^{\perp} A_{\beta}^{\perp}\left(0^{+},-\infty^{-}, \overrightarrow{\boldsymbol{\omega}}_{\perp}\right)\right\} .
\end{aligned}
$$

The transverse components of gluon fields, $A^{\perp}$, have the collinear twist which equals 1 . Therefore, the Wilson line in Eq. (27) represents the infinite amount of the subdominant contributions. Within our frame, it is enough to be limited by the collinear twist three contributions only. In other words, we leave only the terms which include the first order of $A^{\perp}$.

\section{B. The nonstandard hadron tensor (direct process)}

The next step of our consideration is the contribution of the nonstandard diagram, depicted in Fig. 1 (the right panel). The DY hadron tensor receives the contribution from the nonstandard diagram as (before factorization)

$$
\begin{aligned}
\mathcal{W}_{\mu \nu}^{(2)}(g \mid A)= & \int d^{4} k_{1} d^{4} k_{2} \delta^{(4)}\left(k_{1}+k_{2}-q\right) \\
& \times \operatorname{tr}\left[\gamma_{\mu} \mathcal{F}\left(k_{1}\right) \gamma_{\nu} \bar{\Phi}\left(k_{2}\right)\right],
\end{aligned}
$$

where the function $\mathcal{F}\left(k_{1}\right)$ reads

$$
\begin{aligned}
\mathcal{F}\left(k_{1}\right)= & S\left(k_{1}\right) \gamma_{\alpha} \int d^{4} \eta_{1} e^{-i k_{1} \cdot \eta_{1}} \\
& \times\left\langle p_{1}\left|\bar{\psi}\left(\eta_{1}\right) g A_{\alpha}(0) \psi(0)\right| p_{1}\right\rangle .
\end{aligned}
$$

Performing the above-described factorization procedure, the nonstandard hadron tensor takes the following form:

$$
\begin{aligned}
\mathcal{W}_{\mu \nu}^{(2)}(g \mid A)= & \int d x_{1} d y\left[\delta\left(x_{1}-x_{B}\right) \delta\left(y-y_{B}\right)\right] \bar{q}(y) \\
& \times \operatorname{tr}\left[\gamma_{\mu}\left(\int d^{4} k_{1} \delta\left(x_{1} p_{1}^{+}-k_{1}^{+}\right) \mathcal{F}\left(k_{1}\right)\right) \gamma_{\nu} \hat{p}_{2}\right] \\
\equiv & \int d x_{1} d y\left[\delta\left(x_{1}-x_{B}\right) \delta\left(y-y_{B}\right)\right] \\
& \times \bar{q}(y) p_{2}^{-} \mathfrak{N}_{\mu \nu}^{+}\left(x_{1}\right) .
\end{aligned}
$$

We now consider the integral over $k_{1}$ in (30), we write

$$
\begin{aligned}
\mathfrak{N}_{\mu \nu}^{+}= & \int d^{4} k_{1} \delta\left(x_{1} p_{1}^{+}-k_{1}^{+}\right) \\
& \times \operatorname{tr}\left[\gamma_{\mu} \frac{k_{1}^{+} \gamma^{-}+k_{1}^{-} \gamma^{+}-\vec{k}_{1 \perp} \vec{\gamma}_{\perp}}{2 k_{1}^{+} k_{1}^{-}-\vec{k}_{1 \perp}^{2}+i \varepsilon} \gamma_{\alpha} \gamma^{-} \gamma_{\nu} \gamma^{+}\right] \\
& \times \int d^{4} \eta_{1} e^{-i k_{1} \cdot \eta_{1}}\left\langle p_{1}\left|\bar{\psi}\left(\eta_{1}\right) \gamma^{+} g A_{\alpha}(0) \psi(0)\right| p_{1}\right\rangle .
\end{aligned}
$$


Technically, derivation of the longitudinal Wilson line for this case differs from the derivation we implemented for the standard hadron tensor. We notice that for the nonstandard hadron tensor the quark propagator has been included in the soft part.

Let us consider the first term, $k_{1}^{+} \gamma^{-}$, in the quark propagator, see Eq. (31). Thanks to the $\gamma$-structure, this term singles out the $A^{-}$-field in the corresponding correlator. Moreover, the Fourier image of the quark-gluon correlator can be presented in the equivalent form as

$$
\begin{aligned}
& \int d^{4} \eta_{1} e^{-i k_{1} \cdot \eta_{1}-i k_{1} z}\left\langle p_{1}\right| \bar{\psi}\left(\eta_{1}\right) \gamma^{+} \\
& \quad \times\left. g \frac{\partial}{\partial z^{+}} \int_{-\infty^{+}}^{z^{+}} d \omega^{+} A^{-}\left(\omega^{+}, 0^{-}, \overrightarrow{\mathbf{0}}_{\perp}\right)\right|_{z=0} \psi(0)\left|p_{1}\right\rangle,
\end{aligned}
$$

where the derivative with respect to $z^{+}$can be shifted to the exponential function $e^{-i k_{1}^{-} z^{+}}$. As a result, we have

$$
\begin{aligned}
& i k_{1}^{-} \int d^{4} \eta_{1} e^{-i k_{1} \cdot \eta_{1}} \\
& \quad \times\left\langle p_{1}\left|\bar{\psi}\left(\eta_{1}\right) \gamma^{+} g \int_{-\infty^{+}}^{0^{+}} d \omega^{+} A^{-}\left(\omega^{+}, 0^{-}, \overrightarrow{\mathbf{0}}_{\perp}\right) \psi(0)\right| p_{1}\right\rangle .
\end{aligned}
$$

Using Eq. (33), the tensor $\mathfrak{N}_{\mu \nu}$ takes the form of $\left(\vec{k}_{1 \perp}^{2} \approx 0\right)$

$$
\begin{aligned}
\mathfrak{N}_{\mu \nu}^{+}= & \int d^{4} k_{1} \delta\left(x_{1} p_{1}^{+}-k_{1}^{+}\right) \operatorname{tr}\left[\gamma_{\mu} \gamma^{-} \gamma_{\nu} \gamma^{+}\right] \\
& \times \int d^{4} \eta_{1} e^{-i k_{1} \cdot \eta_{1}} \\
& \times\left\langle p_{1}\left|\bar{\psi}\left(\eta_{1}\right) \gamma^{+} i g \int_{-\infty^{+}}^{0^{+}} d \omega^{+} A^{-}\left(\omega^{+}, 0^{-}, \overrightarrow{\mathbf{0}}_{\perp}\right) \psi(0)\right| p_{1}\right\rangle .
\end{aligned}
$$

Thus, the first term finally contributes to the nonstandard part of the hadron tensor as

$$
\begin{aligned}
& \overline{\mathcal{W}}_{\mu \nu}^{(0)}\left(A^{-}\right) \\
& =\int d x_{1} d y\left[\delta\left(x_{1}-x_{B}\right) \delta\left(y-y_{B}\right)\right] \bar{q}(y) \\
& \quad \times \int d^{4} k_{1} \delta\left(x_{1} p_{1}^{+}-k_{1}^{+}\right) \operatorname{tr}\left[\gamma_{\mu} \gamma^{-} \gamma_{\nu} \gamma^{+}\right] \int d^{4} \eta_{1} e^{-i k_{1} \cdot \eta_{1}} \\
& \quad \times\left\langle p_{1}\left|\bar{\psi}\left(\eta_{1}\right) \gamma^{+}\left[-\infty^{+}, 0^{-}, \overrightarrow{\mathbf{0}}_{\perp} ; 0^{+}, 0^{-}, \overrightarrow{\mathbf{0}}_{\perp}\right]_{A^{-}} \psi(0)\right| p_{1}\right\rangle .
\end{aligned}
$$

The exponentiation of $A^{-}$has been presented in Appendix A.

Despite the minus component, $A^{-}$, has formally the collinear twist 2 (the so-called sub-sub-dominant component), the Wilson line with $A^{-}$in Eq. (35) will play the

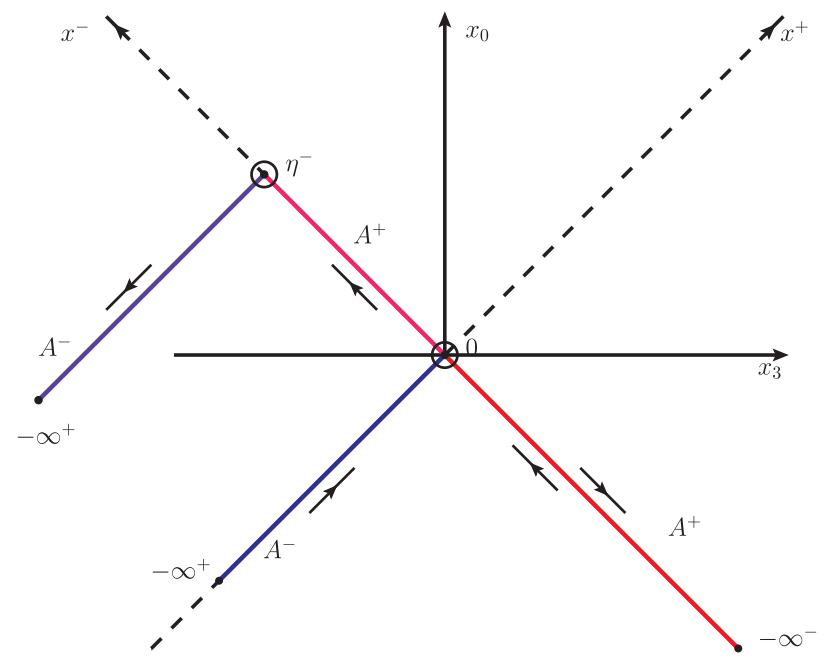

FIG. 2. The longitudinal Wilson lines related to the standard (red lines, the exponentials with $A^{+}$) and nonstandard (blue lines, the exponentials with $A^{-}$) Drell-Yan hadron tensor. The circles single out the interception points which the continuity conditions are defined for.

substantial role for the residual gauge fixing, see the discussion in the next section.

To conclude the section, we restore all the longitudinal Wilson lines which emanate from both the standard and nonstandard hadron tensors, see Fig. 2.

\section{CONTOUR GAUGE: ELIMINATION OF LONGITUDINAL WILSON LINES}

The axial gauge $A^{+}=0$ (as well as the Fock-Schwinger gauges) is in fact a particular case of the most general nonlocal contour gauge determined by a Wilson line with a fixed path. Indeed, the straightforward line in the Wilson exponential which connects $\pm \infty$ with $x$ gives us the axial gauge, while the straightforward line connecting $x_{0}$ with $x$ leads to the Fock-Schwinger gauge. Notice that two different contour gauges can correspond to the same local axial gauge. Meanwhile, to distinguish different contour gauge is very crucial to fix the prescriptions in the gluon poles [15].

In the past, the contour gauge was a very popular subject of intense studies (see, for example, [34]). One of the advantages of using the contour gauge is that the quantum gauge theory becomes free from the Gribov ambiguities. On the other hand, the contour gauge gives the simplest way to fix the gauge including the residual gauge freedom. In contrast to the usual axial gauge, in the contour gauge we first fix an arbitrary point $\left(x_{0}, \mathbf{g}\left(x_{0}\right)\right)$ in the fiber. Then, we define two directions: one of them in the base, the other in the fiber. The direction in the base $\mathbb{R}^{4}$ is nothing else than the tangent vector of a curve which goes through the given point $x_{0}$. The fiber direction can be uniquely determined as the tangent subspace related to the parallel transport. 
Finally, we are able to define uniquely the point in the fiber bundle.

We continue to work with the Drell-Yan hadron tensor derived in [15]. As shown the standard (direct and mirror) diagrams lead to the following Wilson lines in the quarkantiquark nonlocal operator which forms the hadron tensor, see Fig. 2:

$$
\begin{aligned}
& {\left[0^{+}, \eta^{-}, \overrightarrow{\mathbf{0}}_{\perp} ; 0^{+},-\infty^{-}, \overrightarrow{\mathbf{0}}_{\perp}\right]_{A^{+}}, \quad \text { and }} \\
& {\left[0^{+},-\infty^{-}, \overrightarrow{\mathbf{0}}_{\perp} ; 0^{+}, 0^{-}, \overrightarrow{\mathbf{0}}_{\perp}\right]_{A^{+}},}
\end{aligned}
$$

i.e. the gauge invariant quark string operator takes the form of

$$
\begin{aligned}
\bar{\psi}\left(0^{+}, \eta^{-}, \overrightarrow{\mathbf{0}}_{\perp}\right)\left[0^{+}, \eta^{-}, \overrightarrow{\mathbf{0}}_{\perp} ; 0^{+},-\infty^{-}, \overrightarrow{\boldsymbol{0}}_{\perp}\right]_{A^{+}} \Gamma \\
\quad \times\left[0^{+},-\infty^{-}, \overrightarrow{\boldsymbol{0}}_{\perp} ; 0^{+}, 0^{-}, \overrightarrow{\mathbf{0}}_{\perp}\right]_{A^{+}} \psi\left(0^{+}, 0^{-}, \overrightarrow{\mathbf{0}}_{\perp}\right) .
\end{aligned}
$$

Here $\Gamma$ implies a relevant combination of $\gamma$-matrices. The Wilson line (36) is a result of summation in the mirror diagram and the Wilson line (37) appears in the direct diagram.

The sum of direct and mirror diagram contributions takes place if we study the spin-average DY hadron tensor, while, for the single transverse spin asymmetry, we deal individually with only the direct (or mirror) diagram contribution because the direct and mirror diagrams differ in sign to construct the corresponding SSA. For our further considerations in the context of contour gauge, it is not so crucial what kind of hadron tensors we work with.

The nonstandard (direct and mirror) diagrams give us the contributions with the Wilson lines

$$
\begin{aligned}
& {\left[-\infty^{+}, \eta^{-}, \overrightarrow{\mathbf{0}}_{\perp} ; 0^{+}, \eta^{-}, \overrightarrow{\mathbf{0}}_{\perp}\right]_{A^{-}}, \quad \text { and }} \\
& {\left[0^{+}, 0^{-}, \overrightarrow{\mathbf{0}}_{\perp} ;-\infty^{+}, 0^{-}, \overrightarrow{\mathbf{0}}_{\perp}\right]_{A^{-}},}
\end{aligned}
$$

and, therefore, we have the string operator

$$
\begin{aligned}
\bar{\psi}\left(0^{+}, \eta^{-}, \overrightarrow{\boldsymbol{0}}_{\perp}\right)\left[-\infty^{+}, \eta^{-}, \overrightarrow{\mathbf{0}}_{\perp} ; 0^{+}, \eta^{-}, \overrightarrow{\boldsymbol{0}}_{\perp}\right]_{A^{-}} \\
\quad \times \Gamma\left[0^{+}, 0^{-}, \overrightarrow{\mathbf{0}}_{\perp} ;-\infty^{+}, 0^{-}, \overrightarrow{\mathbf{0}}_{\perp}\right]_{A^{-}} \psi\left(0^{+}, 0^{-}, \overrightarrow{\mathbf{0}}_{\perp}\right) .
\end{aligned}
$$

According to the contour gauge conception, we eliminate all the Wilson lines with the longitudinal (unphysical) gluon fields $A^{+}$and $A^{-}$. We note that the ideologically similar approach can be found in [16].

We begin with the Wilson lines shown in Eqs. (36) and (37); we write the following gauge fixing conditions:

$$
\begin{aligned}
& {\left[0^{+}, \eta^{-}, \overrightarrow{\mathbf{0}}_{\perp} ; 0^{+},-\infty^{-}, \overrightarrow{\mathbf{0}}_{\perp}\right]_{A^{+}}=\mathbb{1},} \\
& {\left[0^{+},-\infty^{-}, \overrightarrow{\mathbf{0}}_{\perp} ; 0^{+}, 0^{-}, \overrightarrow{\mathbf{0}}_{\perp}\right]_{A^{+}}=\mathbb{1}}
\end{aligned}
$$

explicit solutions of which read

$$
\begin{aligned}
& A^{+}\left(0^{+}, \mathbb{L}_{0^{-},-\infty^{-}}, \overrightarrow{\mathbf{0}}_{\perp}\right)=0, \\
& A^{+}\left(0^{+}, \mathbb{L}_{-\infty^{-}, \eta^{-}}, \overrightarrow{\mathbf{0}}_{\perp}\right)=0 .
\end{aligned}
$$

Here $\mathbb{L}_{x, y}$ denotes the straightforward line in the Minkowski space connecting point $x$ with point $y$. In the contour gauge (41)-(43), the remaining gluon field components can be represented as (with $\mu=-, \perp$ )

$$
\begin{aligned}
& \left.A_{G}^{\mu}\left(0^{+}, x^{-}, \overrightarrow{\mathbf{0}}_{\perp}\right)\right|_{\text {c.g. }(41)-(43)} \\
& =\int_{-\infty^{-}}^{x^{-}} d z_{\alpha} \frac{\partial z_{\beta}}{\partial x_{\mu}} G^{\alpha \beta}\left(z \mid A_{\text {c.g. }}^{\mu}\right) \\
& =\tilde{n}^{-} \int_{0}^{\infty} d s e^{-\varepsilon s} G^{+\mu}\left(x^{-}-\tilde{n}^{-} s \mid A_{\text {c.g. }}^{\mu}\right)
\end{aligned}
$$

with the boundary condition

$$
\left.A_{\text {b.c. }}^{\mu}\left(0^{+}, x^{-}-\tilde{n}^{-} \infty, \overrightarrow{\boldsymbol{0}}_{\perp}\right)\right|_{\text {c.g. (41)-(43) }}=0 .
$$

In Eq. (44), we use the parametrization of $\mathbb{L}_{-\infty^{-}, x^{-}}$as

$$
\begin{aligned}
z(s) & =\left(0^{+}, x^{-}-\tilde{n}^{-} \lim _{\varepsilon \rightarrow 0} \frac{1-e^{-\varepsilon s}}{\varepsilon}, \overrightarrow{\mathbf{0}}_{\perp}\right), \\
\left.d z_{\alpha}\right|_{x} ^{-\infty} & =\left.\tilde{n}_{\alpha} d s e^{-\varepsilon s}\right|_{0} ^{\infty} .
\end{aligned}
$$

We now dwell on the gauge conditions for $A^{-}$gluon component. We put the Wilson lines (39) to be equal to 1 too, i.e.

$$
\begin{aligned}
& {\left[-\infty^{+}, \eta^{-}, \overrightarrow{\mathbf{0}}_{\perp} ; 0^{+}, \eta^{-}, \overrightarrow{\mathbf{0}}_{\perp}\right]_{A^{-}}=\mathbb{1},} \\
& {\left[0^{+}, 0^{-}, \overrightarrow{\mathbf{0}}_{\perp} ;-\infty^{+}, 0^{-}, \overrightarrow{\mathbf{0}}_{\perp}\right]_{A^{-}}=\mathbb{1} .}
\end{aligned}
$$

These conditions yield

$$
\begin{aligned}
& A^{-}\left(\mathbb{L}_{0^{+},-\infty^{+}}, \eta^{-}, \overrightarrow{\mathbf{0}}_{\perp}\right)=0, \\
& A^{-}\left(\mathbb{L}_{-\infty^{+}, 0^{+}}, 0^{-}, \overrightarrow{\mathbf{0}}_{\perp}\right)=0 .
\end{aligned}
$$

As above, in the contour gauge (47)-(49), the remaining gluon fields have the integral representations which read (here $\mu=+, \perp$ )

$$
\begin{aligned}
& \left.A_{G}^{\mu}\left(x^{+}, \eta^{-}, \overrightarrow{\mathbf{0}}_{\perp}\right)\right|_{\text {c.g. }(47)-(49)} \\
& =\int_{x^{+}}^{-\infty^{+}} d z_{\alpha} \frac{\partial z_{\beta}}{\partial x_{\mu}} G^{\alpha \beta}\left(z \mid A_{\text {c.g. }}^{\mu}\right) \\
& =-\breve{n}^{+} \int_{0}^{\infty} d t e^{-\varepsilon t} G^{-\mu}\left(x^{+}-\breve{n}^{+} t \mid A_{\text {c.g. }}^{\mu}\right)
\end{aligned}
$$

with the boundary condition 


$$
\left.A_{\text {b.c. }}^{\mu}\left(x^{+}-\breve{n}^{+} \infty, \eta^{-}, \overrightarrow{\boldsymbol{0}}_{\perp}\right)\right|_{\text {c.g. (47)-(49) }}=0 .
$$

In Eq. (50) the path parametrization of $\mathbb{L}_{x,-\infty}$ is given by

$$
\begin{aligned}
z(s) & =\left(x^{+}-\breve{n}^{+} \lim _{\varepsilon \rightarrow 0} \frac{1-e^{-\varepsilon t}}{\varepsilon}, \eta^{-}, \overrightarrow{\mathbf{0}}_{\perp}\right), \\
\left.d z_{\alpha}\right|_{x} ^{-\infty} & =-\left.\breve{n}_{\alpha}^{+} d t e^{-\varepsilon t}\right|_{0} ^{\infty} .
\end{aligned}
$$

Further, the gluon field $A_{G}^{-}$of Eq. (44) has to be compatible with the gluon field $A^{-}$of Eq. (48). Also, the same inference has to be valid for the gluon fields $A_{G}^{+}$of Eq. (50) and $A^{+}$of Eq. (43). We thus require the analytical continuity for these gluon fields at the interception points, see Fig. 2, and we finally arrive at the following conditions (here we omit the subscript $G$ ):

$$
\begin{aligned}
& A^{+}\left(0^{+}, x^{-}=\eta^{-}, \overrightarrow{\mathbf{0}}_{\perp}\right)=A^{+}\left(x^{+}=0^{+}, \eta^{-}, \overrightarrow{\mathbf{0}}_{\perp}\right)=0, \\
& A^{-}\left(x^{+}=0^{+}, \eta^{-}, \overrightarrow{\mathbf{0}}_{\perp}\right)=A^{-}\left(0^{+}, x^{-}=\eta^{-}, \overrightarrow{\mathbf{0}}_{\perp}\right)=0,
\end{aligned}
$$

respectively. Having used these conditions, we stay with the physical gluon fields $A^{\perp}$ only.

\section{GLUON PROPAGATOR}

We now go over to consideration of the gluon propagator. In the case of local axial gauge $A^{+}=0$, the gluon propagator is still not a well-defined object because of the spurious singularity related to the residual gauge transformations. In other words, the axial gauge cannot fix completely the unique element of each orbit defined on the gauge group. In Appendix B, we present the handbook material regarding the gauge and residual gauge fixing. It is clear that if, in the local axial gauge $A^{+}=0$, we fix the residual gauge by requiring $\theta_{0}^{a}\left(k^{-}, \overrightarrow{\mathbf{k}}_{\perp}\right)=0$ [see Eqs. (B32)-(B37)] we immediately get that $A^{-}=0$ as well. The same inference can be reached in the simplest way if we use the contour gauge conception [see Eq. (53)]. Notice that the maximal gauge fixing which is based on the contour gauge conception does not relate technically to the problem of finding the inverse kinematical operator [see Eqs. (B45)-(B50)]. The contour gauge approach is, therefore, an alternative method of gauge fixing compared to the "classical" approaches based on the corresponding effective Lagrangian (see, for example, [17]).

So, we perform our calculation in the contour gauge defined by Eqs. (41) and/or (47) together with the conditions of Eq. (53) where the only physical gluons are presented. In the framework of collinear factorization under our consideration, the gluon momentum has the plus dominant components.

Having used the Wilson lines from the standard and nonstandard diagrams, we calculate the gluon propagator which reads $\left\langle 0\left|T A_{\perp}^{\mu}\left(0^{+}, x^{-}, \overrightarrow{\mathbf{0}}_{\perp}\right) A_{\perp}^{\nu}\left(0^{+}, 0^{-}, \overrightarrow{\mathbf{0}}_{\perp}\right)\right| 0\right\rangle=D_{\perp}^{\mu \nu}\left(x^{-}\right)$.

Using the integral representation (44), the gluon propagator takes the form of

$$
\begin{aligned}
D_{\perp}^{\mu \nu}\left(x^{-}\right)= & n_{\alpha} n_{\beta} \int_{0}^{\infty} d s d s^{\prime} e^{-\varepsilon s-\varepsilon s^{\prime}} \\
& \times\left\langle 0\left|T G^{\mu \alpha}\left(x^{-}-\tilde{n}^{-} s\right) G^{\nu \beta}\left(0^{-}-\tilde{n}^{-} s^{\prime}\right)\right| 0\right\rangle \\
= & \int\left(d^{4} \ell\right) e^{-i \ell^{+} x^{-}} \frac{1}{\ell^{2}+i 0} \frac{\left(\ell^{+}\right)^{2} d_{\perp}^{\mu \nu}(\ell)}{\left(\ell^{+}+i \varepsilon\right)\left(\ell^{+}-i \varepsilon\right)} .
\end{aligned}
$$

In Eq. (55), we have explicitly performed the integration over $d s\left(d s^{\prime}\right)$ :

$$
\int_{0}^{\infty} d s e^{ \pm i s\left(\ell^{+} \pm i \varepsilon\right)}=\frac{ \pm i}{\ell^{+} \pm i \varepsilon}
$$

which emanates from the path parametrization. It is worth emphasizing the gluon pole prescription can be traced from this kind of integrations (see [15]). The transverse tensor $d_{\perp}^{\mu \nu}$ has been constructed as

$$
d_{\perp}^{\mu \nu}(\ell)=g^{\mu \nu}-\frac{\ell^{\mu,+} n^{\nu}+\ell^{\nu,+} n^{\mu}}{\left[\ell^{+}\right]_{\mathrm{reg}}},
$$

where the spurious singularity $\left[\ell^{+}\right]_{\text {reg }}$ has to be regularized.

We consider the combination

$$
\frac{\left(\ell^{+}\right)^{2}}{\left(\ell^{+}+i \varepsilon\right)\left(\ell^{+}-i \varepsilon\right)} d_{\perp}^{\mu \nu}(\ell) .
$$

The first term of Eq. (58) includes the combination

$$
g^{\mu \nu} \ell^{+} \frac{\ell^{+}}{\left(\ell^{+}+i \varepsilon\right)\left(\ell^{+}-i \varepsilon\right)}
$$

which has to be treated only as

$$
g^{\mu \nu} \frac{\ell^{+}}{2}\left(\frac{1}{\ell^{+}+i \varepsilon}+\frac{1}{\ell^{+}-i \varepsilon}\right)=g^{\mu \nu} \ell^{+} \frac{\mathcal{P}}{\ell^{+}}=g^{\mu \nu} .
$$

On the other hand, for $x^{-}>0$ [see the momentum integral (55)], the integration contour has to be closed in the lower semiplane, $\Im \mathrm{m} \ell^{+}<0$. Hence, for the $g_{\mu \nu}$-term, we obtain the integrand

$$
g^{\mu \nu} \frac{\ell^{+}}{\ell^{+}+i \varepsilon},
$$

where the denominator $\ell^{+}-i \varepsilon$ has been canceled by one of $\ell^{+}$in the numerator. It is clear that the remaining combination in Eq. (61) yields $g_{\mu \nu}$ [cf. Eq. (60)]. 
Regarding the second term of Eq. (58), we propose two ways of reasoning.

\section{A. The first way}

We do not specify explicitly the tensor structure of this term. The second term of Eq. (58) can be written in the following form (here the momentum flux direction is not fixed):

$\frac{\left(\ell^{+}\right)^{2}}{\left(\ell^{+}+i \varepsilon\right)\left(\ell^{+}-i \varepsilon\right)} \frac{L^{\mu \nu}(\ell, n)}{\left[\ell^{+}\right]_{\mathrm{reg}}}=\ell^{+} \frac{\mathcal{P}}{\ell^{+}} \frac{L^{\mu \nu}(\ell, n)}{\left[\ell^{+}\right]_{\mathrm{reg}}}$,

where we use

$$
\frac{\mathcal{P}}{\ell^{+}}=\frac{\ell^{+}}{\left(\ell^{+}+i \varepsilon\right)\left(\ell^{+}-i \varepsilon\right)} .
$$

To well-define the product of two generalized functions the pole $1 /\left[\ell^{+}\right]_{\text {reg }}$ must be treated only as

$$
\frac{1}{\left[\ell^{+}\right]_{\mathrm{reg}}}=\frac{\mathcal{P}}{\ell^{+}} \text {. }
$$

Indeed, we have

$$
\frac{\mathcal{P}}{\ell^{+}} \ell^{+} \frac{\mathcal{P}}{\ell^{+}}=\frac{\mathcal{P}}{\ell^{+}} .
$$

On the other hand, if we let $1 /\left[\ell^{+}\right]_{\text {reg }}$ be equal to $1 /\left(\ell^{+} \pm i \varepsilon\right)$, we will face on the wrong-defined product of two generalized functions [35]:

$$
\begin{aligned}
\frac{\mathcal{P}}{\ell^{+}} \ell^{+} \frac{1}{\ell^{+} \pm i \varepsilon} & =\frac{\mathcal{P}}{\ell^{+}} \ell^{+}\left(\frac{\mathcal{P}}{\ell^{+}} \mp i \pi \delta\left(\ell^{+}\right)\right) \\
\Rightarrow & \frac{\mathcal{P}}{\ell^{+}} \ell^{+} \delta\left(\ell^{+}\right) \\
& \text {- wrong-defined product. }
\end{aligned}
$$

\section{B. The second way}

We take into account that the tensor structure includes the plus component of the gluon momentum. Hence, the second term of Eq. (58) reads

$$
\frac{\left(\ell^{+}\right)^{2}}{\left(\ell^{+}+i \varepsilon\right)\left(\ell^{+}-i \varepsilon\right)} \frac{\ell^{\mu,+} n^{\nu}+\ell^{\nu,+} n^{\mu}}{\left[\ell^{+}\right]_{\mathrm{reg}}} .
$$

Here, as shown above, for the first factor, we can again use that

$$
\frac{\left(\ell^{+}\right)^{2}}{\left(\ell^{+}+i \varepsilon\right)\left(\ell^{+}-i \varepsilon\right)}=\ell^{+} \frac{\mathcal{P}}{\ell^{+}}=1
$$

and, for the second factor, we have

$$
\frac{\ell^{\mu,+} n^{\nu}+\ell^{\nu,+} n^{\mu}}{\left[\ell^{+}\right]_{\mathrm{reg}}}=\frac{\ell^{+}}{\left[\ell^{+}\right]_{\mathrm{reg}}}\left(n^{* \mu} n^{\nu}+n^{* \nu} n^{\mu}\right) .
$$

Based on this expression, it is clear that the only possibility is to define $1 /\left[\ell^{+}\right]_{\text {reg }}$ through the principle value, see Eq. (64).

Thus, in the contour gauge generated by both the standard and nonstandard diagrams, the gluon propagator reads

$$
\begin{aligned}
& D_{\perp}^{\mu \nu}\left(x^{-}\right) \\
& =\int\left(d^{4} \ell\right) e^{-i \ell^{+} x^{-}} \frac{1}{\ell^{2}+i 0}\left\{g^{\mu \nu}-\frac{\mathcal{P}}{\ell^{+}}\left(\ell^{\mu,+} n^{\nu}+\ell^{\nu,+} n^{\mu}\right)\right\}
\end{aligned}
$$

or, using Eq. (68), we obtain

$$
D_{\perp}^{\mu \nu}\left(x^{-}\right)=\int\left(d^{4} \ell\right) e^{-i \ell^{+} x^{-}} \frac{g_{\perp}^{\mu \nu}}{\ell^{2}+i 0},
$$

where $g_{\perp}^{\mu \nu}=g^{\mu \nu}-n^{* \mu} n^{\nu}-n^{* \nu} n^{\mu}$.

We notice that the gluon propagator presented in Eq. (71) takes place for the very specific case of the polarized DY hadron tensor under our consideration. In the case of deep-inelastic scattering process, where the corresponding Wilson lines are different, the gluon propagator derived in the contour gauge frame has the form similar to Eq. (73), see below. We also stress that, in Eqs. (70) and (71), the gluon momentum flux is not important and is not specified.

We now consider a particular case wherein only the standard diagram exists. For example, this can be achieved if we neglect the higher twist correlators $\left\langle\bar{\psi} A^{-} \psi\right\rangle$ which appear in the nonstandard diagram. Moreover, the gluon field coordinates are not necessarily on the minus direction and the gluon momentum flux is fixed in the positive direction from the $\nu$-vertex to $\mu$-vertex. In this case, the gluon propagator reads

$$
\begin{aligned}
& \left.D^{\mu \nu}(x)\right|_{\text {fixed flux }} ^{\text {stand dia. }} \\
& =\int\left(d^{4} \ell\right) e^{-i \ell x} \frac{1}{\ell^{2}+i 0} \\
& \times\left\{g^{\mu \nu}-\frac{\mathcal{P}}{\ell^{+}}\left(\ell^{\mu} n^{\nu} \theta\left(\ell^{+}\right)+\ell^{\nu} n^{\mu} \theta\left(-\ell^{+}\right)\right)\right\},
\end{aligned}
$$

where the corresponding $\theta$-functions specify the momentum flux. Using the Cauchy theorem in Eq. (72), we finally arrive at

$$
\begin{aligned}
\left.D^{\mu \nu}(x)\right|_{\text {fixed flux }} ^{\text {stand.ia. }}= & \int\left(d^{4} \ell\right) \frac{e^{-i \ell x}}{\ell^{2}+i 0} \\
& \times\left\{g^{\mu \nu}-\frac{\ell^{\mu} n^{\nu}}{\ell^{+}-i \varepsilon}-\frac{\ell^{\nu} n^{\mu}}{\ell^{+}+i \varepsilon}\right\}
\end{aligned}
$$


which coincides with the results in $[16,17]$. This expression is sensitive to the definition of the positive (negative) flux direction [see Eq. (72)]. Hence, the symmetry over $\mu \leftrightarrow \nu$ takes place only together with the simultaneous replacement $\ell^{+} \leftrightarrow-\ell^{+}$in the second and third terms of Eq. (73).

\section{CONCLUSIONS AND DISCUSSIONS}

In the contour gauge, from the technical viewpoint, the maximal gauge fixing is not associated with the problem of finding the inverse kinematical operator. Hence, the contour gauge approach has to be considered as the alternative method of gauge fixing in comparison with the classical approaches based on the corresponding effective Lagrangians. It is necessary to stress that the contour gauge contains the important and unique additional information (needed to fix the prescription in the gluon poles) which is invisible in the case of usual (local) axial gauge. From this point of view, before we discard the terms with $A^{+}$, we have to determine the relevant fixed path in the corresponding Wilson line with $A^{+}$which finally leads to certain prescriptions in the gluon poles. Moreover, the corresponding Wilson line with $A^{-}$in the nonstandard diagram, which contributes to the polarized DY hadron tensor, prompts the way of residual gauge fixing.

We thus advocate the preponderance of the contour gauge use which allows to fix completely the gauge freedom by the most illustrative and simplest way. We demonstrate that the nonstandard diagram plays the important role in forming the relevant contour in the corresponding Wilson line. Hence, from the viewpoint of contour gauge, there is no way to neglect the additional nonstandard diagram.

\section{ACKNOWLEDGMENTS}

We thank A. V. Efremov, A. Prokudin and L. Szymanowski for useful discussions. The work by I. V. A. was partially supported by the HeisenbergLandau Program of the German Research Foundation (DFG). I. V. A. also thanks the Department of Physics at the University of Antwerpen for warm hospitality.

\section{APPENDIX A: EXPONENTIATION OF COMPONENT $A^{-}$}

In this Appendix, we demonstrate the method of the $A^{-}$ component exponentiation. In fact, there are several methods how to exponentiate the gluon fields, see e.g. [36-38]. Here, we present an alternative frame-independent and most efficient method mainly based on the approach described in [32], see Sec. 46.

\section{Some conventional notations}

Before going further, we remind of several conventions regarding how the gauge transformations match the
Wilson lines. Taking, for the sake of simplicity, the Abelian gauge theory (in the case of interest the distinction between Abelian and non-Abelian groups is irrelevant), let us assume that the fermion and gauge fields are transformed as

$$
\begin{gathered}
\psi^{\omega}(x)=e^{ \pm i \theta(x)} \psi(x), \\
A_{\mu}^{\omega}(x)=A_{\mu}(x) \pm \partial_{\mu} \theta(x),
\end{gathered}
$$

where $\omega$ stands here for the gauge transformation. Generally speaking, the signs at the gauge function $\theta$ in Eqs. (A1) and (A2) are conventional. If we fix the transformations as in Eqs. (A1) and (A2), i.e. the same signs in both expressions, then we can readily see that the covariant derivative and the gauge-invariant fermion string operator become

$$
\begin{aligned}
i \mathcal{D}_{\mu} & =i \partial_{\mu}+g A_{\mu}(x), \\
\mathbb{O}^{\text {g.inv. }}(x, y) & =\bar{\psi}(y)[y ; x]_{A} \psi(x),
\end{aligned}
$$

where the Wilson line is given by

$$
\begin{aligned}
{[y ; x]_{A} \stackrel{\text { def }}{=} } & \mathbb{P} \exp \left\{+i g \int_{x}^{y} d z_{\mu} A^{\mu}(z)\right\} \\
= & \lim _{N \rightarrow \infty}\left[y ; x_{N}\right]_{A}\left[x_{N} ; x_{N-1}\right]_{A} \cdots\left[x_{1} ; x\right]_{A} \\
= & \lim _{N \rightarrow \infty}\left[1+i g A\left(x_{N}\right) \cdot\left(y-x_{N}\right)\right] \\
& \cdots\left[1+i g A(x) \cdot\left(x_{1}-x\right)\right] .
\end{aligned}
$$

In Eq. (A4), the starting point $x$ and final point $y$ are connected by the certain path $\mathbb{P} \in \mathbb{R}^{4}$ which allows the arrangement by pounding $\left\{x_{N}\right\}_{x}^{y}$.

However, if the signs in both fermion and gauge boson transformations differ from each other, i.e.

$$
\begin{aligned}
& \psi^{\omega}(x)=e^{ \pm i \theta(x)} \psi(x), \\
& A_{\mu}^{\omega}(x)=A_{\mu}(x) \mp \partial_{\mu} \theta(x),
\end{aligned}
$$

the covariant derivative takes the form of

$$
i \mathcal{D}_{\mu}=i \partial_{\mu}-g A_{\mu}(x),
$$

while the gauge-invariant fermion string operator, in this case, reads (see, for example, [16])

$$
\mathbb{O}^{\text {g.-inv. }}(x, y)=\bar{\psi}(y)[x ; y]_{A} \psi(x)
$$

or

$$
\mathbb{O}^{\text {g.inv. }}(x, y)=\bar{\psi}(y)[y ; x]_{A}^{-1} \psi(x)
$$

with the Wilson line defined as in Eq. (A4).

In our paper, we adhere to the conventions as in Eq. (A3). 


\section{Description of the method}

We begin with the most illustrative subject which is the Green function in the external field. The gluon radiation from the proper spinor line as shown in Fig. 1 (right panel) is actually relevant to the Green function in the external field.

Consider the differential equation for the Green function

$$
[i \hat{\partial}+g \hat{A}(x)] \mathrm{S}(x, y)=-\delta^{(4)}(x-y),
$$

where the wide hat denotes the convolution with $\gamma$-matrices as $\hat{A}=\gamma \cdot A$ etc.

We emphasize that the Green function defined by Eq. (A10) is not a gauge-invariant subject (see, for example, [36,37]). As one can see below, namely the gauge-noninvariant Green function ensures the appearance of the gauge-invariant fermion string operator in the corresponding hadron matrix element.

For the sake of simplicity and without the loss of generality, we assume that $\partial^{\mu}=\left(0^{+}, \partial^{-}, \overrightarrow{\mathbf{0}}_{\perp}\right), A^{\mu}=$ $\left(0^{+}, A^{-}, \overrightarrow{\mathbf{0}}_{\perp}\right)$ and we, therefore, study the tensor combination as $\mathrm{S}^{\left[\gamma^{+}\right]}(x, y) \stackrel{\text { def }}{=} \gamma^{+} \mathrm{S}(x, y)$. That is, instead of Eq. (A10) we deal with the following differential equation:

$$
\left[i \partial^{-}+g A^{-}(x)\right] \mathrm{S}^{\left[\gamma^{+}\right]}(x, y)=-\delta^{(4)}(x-y) .
$$

Hence, in the operator forms, the Green function takes the form of

$$
\mathrm{S}^{\left[\gamma^{+}\right]}(x, y)=-\frac{1}{\left[i \hat{\partial}^{-}+g \hat{A}^{-}(x)\right]} \delta^{(4)}(x-y),
$$

where the small hat now denotes the corresponding operators. From the mathematical point of view, the inverse operator is defined via the integral representation as

$$
\frac{i}{\left[i \hat{\partial}^{-}+g \hat{A}^{-}(x)\right]}=\lim _{\varepsilon \rightarrow 0} \int_{0}^{\infty} d \nu e^{i \nu\left[i \hat{\partial}^{-}+g \hat{A}^{-}(x)+i \varepsilon\right]} .
$$

Hence, we can write the Green function as

$$
\begin{aligned}
\mathrm{S}^{\left[\gamma^{+}\right]}(x, y) & =i \int_{0}^{\infty} d \nu e^{i \nu\left[i \hat{\partial}^{-}+g \hat{A}^{-}(x)+i \varepsilon\right]} \delta^{(4)}(x-y) \\
& \equiv i \int_{0}^{\infty} d \nu \mathcal{U}(\nu) .
\end{aligned}
$$

Here and in what follows the limit symbol has been omitted. In the momentum representation, $\mathcal{U}(\nu)$ takes the form of

$$
\mathcal{U}(\nu)=\int\left(d^{4} p\right) e^{-i p(x-y)+i \nu \hat{p}+i \mathcal{K}(x, \nu)-\varepsilon \nu},
$$

where the integration measure $\left(d^{4} p\right)$ includes all needed normalization constants and we use

$$
e^{-\nu \hat{\partial}^{-}} e^{-i p(x-y)}=e^{-i p(x-y)} e^{i \nu \hat{p}^{-}}
$$

which defines how the operator acts. In Eq. (A15), the function $\mathcal{K}(x, \nu)$ is an unknown function which we have to derive.

Since the function $\mathcal{U}(\nu)$ obeys (we can check that by straightforward calculations)

$$
-i \frac{\partial \mathcal{U}(\nu)}{\partial \nu}=\left[i \hat{\partial}^{-}+g \hat{A}^{-}(x)+i \varepsilon\right] \mathcal{U}(\nu),
$$

the function $\mathcal{K}(x, \nu)$ has to satisfy the following differential equation:

$$
\frac{\partial \mathcal{K}(x, \nu)}{\partial \nu}=-\partial^{-} \mathcal{K}(x, \nu)+g A^{-}(x)
$$

provided $\mathcal{K}(x, \nu=0)=0$. A solution of Eq. (A18) can be easily found (see [32]); it reads

$$
\begin{aligned}
\mathcal{K}(x, \nu) & =g \int_{0}^{\nu} d s \int\left(d^{4} k\right) e^{-i k\left(x-s \breve{n}^{+}\right)} A^{-}(k) \\
& =g \int_{0}^{\nu} d s A^{-}\left(x-s \breve{n}^{+}\right) .
\end{aligned}
$$

Using Eq. (A19), the corresponding Green function takes the form of

$$
\begin{aligned}
\mathrm{S}^{\left[\gamma^{+}\right]}(x, y)= & i \int_{0}^{\infty} d \nu e^{-\varepsilon \nu} \delta^{(4)}\left(x-y-\nu \breve{n}^{+}\right) \\
& \times \exp \left\{-i g \int_{x}^{y} d z^{+} A^{-}\left(z^{+}\right)\right\}
\end{aligned}
$$

where the standard integral representation for $\delta$-function has been used,

$$
\delta^{(4)}\left(x-y-\nu \breve{n}^{+}\right)=\int\left(d^{4} p\right) e^{-i p(x-y)+i \nu p},
$$

and we trade $x-\nu \breve{n}^{+}$(see the upper integral limit in integration over $d z^{+}$) for $y$ thanks to the argument of $\delta$-function.

The final stage is to write the integration of the $\delta$-function as

$$
\begin{aligned}
& i \int_{0}^{\infty} d \nu e^{-\varepsilon \nu} \delta^{(4)}\left(x-y-\nu \breve{n}^{+}\right) \\
& =i \int_{0}^{\infty} d \nu e^{-\nu \hat{\partial}^{-}-\varepsilon \nu} \delta^{(4)}(x-y) \\
& =-\frac{1}{\left[i \hat{\partial}^{-}+i \varepsilon\right]} \delta^{(4)}(x-y) \equiv S^{c\left[\gamma^{+}\right]}(x-y) .
\end{aligned}
$$

Thus, we derive that 


$$
\mathbf{S}^{\left[\gamma^{+}\right]}(x, y)=S^{c\left[\gamma^{+}\right]}(x-y)[x ; y]_{A^{-}},
$$

where $S^{c}(x-y)$ is defined through $\langle 0|T \psi(x) \bar{\psi}(y)| 0\rangle$ and we use the obvious property $[x ; y]_{A}=[y ; x]_{A}^{-1}$.

The extension to the non-Abelian gauge group is straightforward.

From Eq. (A23), we can conclude that the fermion field operator in the external field reads

$$
\Psi\left(x^{+} \mid A\right)=\psi\left(x^{+}\right) \exp \left\{i g \int_{\mathbb{C}}^{x^{+}} d z^{+} A^{-}\left(z^{+}\right)\right\},
$$

where $\mathbb{C}$ is, in principle, an arbitrary point which however we choose to be equal to $-\infty^{+}$.

We stress that the fermion in the external field differs from the gauge-invariant fermion field which appears in the string operator, see Eq. (A3). Indeed, as is well known (see, for example, [37,38]) in order to get the gauge-invariant string operator it is necessary to include the gauge boson (gluon) radiations from the fermions after the interaction of them with photons (or other gauge bosons) as shown in Fig. 1 (left panel). Otherwise, we deal with the fermions in the external fields which are not gauge invariant [see Fig. 1 (right panel)].

To illustrate the last statement, let us consider the simplest case of Compton-like amplitude (see also [38]). We have

$$
T^{\mu \nu}=\int\left(d^{4} x\right) e^{-i q \cdot x}\left\langle p\left|T J^{\mu}(x) J^{\nu}(0)\right| p\right\rangle .
$$

On the handbag diagram level, we have

$$
\begin{aligned}
T^{\mu \nu}= & \int\left(d^{4} x\right) e^{-i q \cdot x} \\
& \times\left\langle p\left|: \bar{\psi}(x) \gamma^{\mu} \psi(x) \bar{\psi}(0) \gamma^{\nu} \psi(0):\right| p\right\rangle .
\end{aligned}
$$

In order to include all gauge boson radiations from the fermion propagator given by the fermion contraction, we merely make a substitution (modulo the conventional normalizations which are now irrelevant):

$$
\overrightarrow{\psi(x) \bar{\psi}}(0)=S^{c}(x) \Rightarrow \mathrm{S}(x, 0)
$$

with $\mathrm{S}(x, 0)$ being a gauge-noninvariant Green function, see Eq. (A23). Using the relation which is similar to Eq. (A23), we can obtain that

$$
\begin{aligned}
T^{\mu \nu}= & \int\left(d^{4} x\right) e^{-i q \cdot x} \\
& \times\left\langle p\left|: \bar{\psi}(x) \gamma^{\mu} S^{c}(x)[x ; 0]_{A} \gamma^{\nu} \psi(0):\right| p\right\rangle .
\end{aligned}
$$

After the factorization procedure, the matrix combination $\gamma^{\mu} S^{c} \gamma^{\nu}$ refers to the so-called hard part, while the nonperturbative hadron matrix element involves the gauge-invariant string operator defined as

$$
\left\langle p\left|: \bar{\psi}(x)[x ; 0]_{A} \psi(0):\right| p\right\rangle \text {. }
$$

\section{APPENDIX B: GAUGE AND RESIDUAL GAUGE SYMMETRIES}

In this Appendix, we remind of some subtleties related to the residual gauge transformations in different gauge theories.

\section{Classical $\boldsymbol{U}(\mathbf{1})$-gauge theory (Abelian theory)}

The $U(1)$-gauge theory, where the gauge transformation

$$
A_{\mu}^{\Lambda}(x)=A_{\mu}(x)+\partial_{\mu} \Lambda(x),
$$

defines an orbit on the $U(1)$-group. In the Abelian case, the strength tensor $F_{\mu \nu}$ is gauge invariant and, therefore, only the longitudinal (unphysical) components of the field, $A_{\mu}^{L}$, can be gauge transformed. Indeed, in the classical gauge theory for both $k^{2}=0$ and $k^{2} \neq 0$, the solution of the Maxwell equation in vacuum, $\partial_{\mu} F_{\mu \nu}=0$, reads (modulo the complex conjugated terms) (see, e.g., [39])

$$
\begin{aligned}
A_{\mu}(x)= & A_{\mu}^{L}(x)+A_{\mu}^{\perp}(x) \\
= & \int\left(d^{4} k\right) e^{i k x} k_{\mu} a_{L}(k) \\
& +\int\left(d^{4} k\right) e^{i k x} \delta\left(k^{2}\right) e_{\mu}^{\perp(\alpha)} a_{\perp}^{(\alpha)}(k),
\end{aligned}
$$

where $\left(d^{4} k\right)$ stands for the corresponding integration measure with an appropriate normalization, $\alpha=(1,2)$ and $k \cdot e^{\perp(\alpha)}=0$. With this expression, we can easily derive the gauge transformations in $p$-space $(=$ the momentum representation)

$k_{\mu} a_{L}^{\Lambda}(k)+e_{\mu}^{\perp} a_{\perp}^{\Lambda}(k)=k_{\mu} a_{L}(k)+e_{\mu}^{\perp} a_{\perp}(k)+k_{\mu} \tilde{\Lambda}(k),(\mathrm{B} 3$

where the imaginary factor $i$ is absorbed in the definition of $\tilde{\Lambda}$. In what follows summation over $\alpha$ and dimensionful normalizations are not shown explicitly unless it leads to misunderstanding.

Since $k \cdot e^{\perp(\alpha)}=0$, we conclude that

$$
a_{L}^{\Lambda}(k)=a_{L}(k)+\tilde{\Lambda}(k), \quad a_{\perp}^{\Lambda}(k)=a_{\perp}(k),
$$

or, equivalently,

$$
\begin{aligned}
& A_{\mu}^{L, \Lambda}(k)=A_{\mu}^{L}(k)+k_{\mu} \tilde{\Lambda}(k), \\
& A_{\mu}^{\perp, \Lambda}(k)=A_{\mu}^{\perp}(k) .
\end{aligned}
$$

Moreover, it is easy to demonstrate that 


$$
A_{\mu}^{L, \Lambda}(x)=-i \partial_{\mu} \alpha(x),
$$

where $\alpha(x)$ is a scalar function which is related to $a_{L}(k)$ via the Fourier transformation, $\alpha(x) \stackrel{\mathrm{F}}{=} a_{L}(k)$, and $a_{L}(k)=$ $\xi(k) / k^{2}$ with $\xi(k) \stackrel{\text { def }}{=} k \cdot A^{L}(k) \neq 0$ for $k^{2} \neq 0$. Notice that if $k^{2}=0$, the Maxwell equation takes the simplest form, $k \cdot A(k)=0$, in the $p$-space and, therefore, $k \cdot A^{L}(k)=$ $k^{2} a_{L}(k)=0$ or, in other words, $a_{L}(k)=\xi(k) / k^{2} \sim 0 / 0$.

As is well known, to fix the certain representative on the group orbit we have to impose a gauge condition $F\left(A^{\Lambda}\right)=$ 0 on the gauge-transformed fields in order to find a solution with respect to the gauge parameter $\Lambda$. Here, we do not discuss the appearance of Gribov's ambiguity.

\section{a. The Lorentz gauge}

As the first example, we consider the Lorentz (covariant) condition which states

$$
\partial_{\mu} A_{\mu}^{\Lambda}(x)=\partial_{\mu} A_{\mu}+\partial^{2} \Lambda(x)=0 .
$$

In $p$-space, condition (B7) takes the following form:

$$
k_{\mu} A_{\mu}^{L}(k)+k^{2} \tilde{\Lambda}(k)=0
$$

which gives us the relation $a_{L}(k)=-\tilde{\Lambda}(k)$ for the case of $k^{2} \neq 0$. Notice that if $k^{2}=0$, then the functions $a_{L}(k)$ and $\tilde{\Lambda}(k)$ in the combination $a_{L}(k)+\tilde{\Lambda}(k)$ are free functions and they are independent of each other.

However, the gauge condition (B7) [or (B8)] cannot fix the orbit representative uniquely. Indeed, there is still the so-called residual gauge freedom defined by $F\left(A^{\Lambda}\right)=F(A)=0$. For the Lorentz condition, two simultaneous conditions,

$$
\partial_{\mu} A_{\mu}^{\Lambda}(x)=0 \quad \text { and } \quad \partial_{\mu} A_{\mu}(x)=0,
$$

lead to

$$
\partial^{2} \Lambda_{0}(x)=0
$$

where the gauge function (parameter) $\Lambda_{0}$ defines the residual gauge freedom. That is, the residual gauge transformation with the function $\Lambda_{0}$ keeps the gauge condition, $F(A)=0$, gauge invariant. Hence, the gauge freedom fixing means that one fixes all gauge freedom including the residual gauge. In other words, if there is no residual gauge transformation, the given gauge condition fixes the gauge freedom completely and we deal with one representative on a gauge orbit.

Let us consider the second gauge condition in Eq. (B9). In $p$-space, it leads to the following possibilities $\left(k \cdot a_{\perp}=0\right.$ by definition):

$$
k^{2} a_{L}(k)=0 \Rightarrow \begin{cases}k^{2}=0, & a_{L}(\vec{k})-\text { arbitrary } \\ k^{2} \neq 0, & a_{L}(k)=0 .\end{cases}
$$

Hence, we can see that the gauge condition (B9) cannot eliminate the unphysical field $A_{\mu}^{L}$ for the case of $k^{2}=0$. Working with Eq. (B10), in the same manner, we conclude that the gauge function $\tilde{\Lambda}_{0}(\vec{k})$ is not fixed and generates the residual gauge transformation provided $k^{2}=0$.

It is instructive to consider the condition (B10) in the coordinate representation ( $x$-space). Solution (B10) can be easily found and represented, for instance, in the following form:

$$
\Lambda_{0}(x)= \begin{cases}\text { const } & \\ 1 / x^{2}, & \text { for } x^{2} \neq 0 \\ C_{0} e^{i\left(x_{0}-\vec{x} \vec{N}\right)} & \text { with, }|\vec{N}|=1\end{cases}
$$

Notice that the scalar function $\alpha(x)$ in Eq. (B6) which obeys the second condition in Eq. (B9), i.e. $\partial^{2} \alpha(x)=0$, has formally the same form as (B12).

For $k^{2} \neq 0$, the scalar gauge function $\Lambda$ gives also the longitudinal (unphysical) field $A_{\mu}^{L}$, see (B8). Therefore, the first two solutions of (B12) are irrelevant for our study. In order to get matched with the corresponding condition (B11) in the momentum representation, we have to put $C_{0}$ equal to zero, $C_{0}=0$. However, for the case of $k^{2}=0$, as mentioned above, the functions $\alpha(x)$ and $\Lambda_{0}(x)$ are independent and arbitrary due to the different free constant prefactors in the plane wave solution.

We can also consider the Lorentz gauge condition (B7) as an inhomogeneous differential equation with respect to $\Lambda(x)$, i.e.

$$
\partial^{2} \Lambda(x)=\eta(x),
$$

where $\eta(x) \stackrel{\text { def }}{=}-\partial_{\mu} A_{\mu}(x)$. Solving (B13), we obtain that

$$
\Lambda(x)=\Lambda_{0}(x)+\int d^{4} y G(x-y) \eta(y),
$$

where the Green function $G(x)$ is defined as

$$
G(x)=\frac{1}{\left[\partial^{2}\right]_{\mathrm{reg}}} \delta^{(4)}(x)
$$

with the suitable regularization of operator stemmed from the corresponding boundary conditions, see [32].

\section{B. The Coulomb gauge}

Using the condition $A_{0}^{\Lambda}(x)=0$ to amplify the Lorentz condition (B7), we can get the Coulomb gauge condition which reads 


$$
\vec{\partial} \vec{A}^{\Lambda}(x)=\vec{\partial} \vec{A}(x)+\Delta \Lambda(x)=0 .
$$

In $p$-space, the condition (B16) is transformed to (recall that $\vec{\partial} \vec{A}^{\perp}=0$ by construction)

$$
\vec{k}^{2} a_{L}(k)+\vec{k}^{2} \tilde{\Lambda}(k)=0 .
$$

Again, let us study the corresponding residual gauge freedom:

$$
\vec{\partial} \vec{A}(x)=0 \quad \text { and } \quad \Delta \Lambda(x)=0 .
$$

For the sake of simplicity, we dwell on the case of $k^{2}=0$ which leads to $\vec{k}^{2} \neq 0$. With this, instead of (B17), it is enough to stop on the equation

$$
\vec{k}^{2} \tilde{\Lambda}(\vec{k})=0
$$

Hence, the only solution of (B19) is $\tilde{\Lambda}=0$ which means that there is no residual freedom at all.

Therefore, in the Coulomb gauge there are no longitudinal field components and we deal with the physical gauge field $A_{\mu}^{\perp}$ only.

\section{c. The Hamilton and axial gauges}

In a similar manner, we can study the residual gauge symmetries in the Hamilton $\left(A_{0}^{\Lambda}=0\right)$ and axial $\left(A^{+, \Lambda}=0\right)$ gauges. The residual gauge transformations are given by the corresponding free (unfixed) gauge function $\tilde{\Lambda}(k)$ provided $k_{0}=0$ or $k^{+}=0$.

\section{Classical $S U(3)$-gauge theory (non-Abelian theory)}

The next subject of our discussion is a non-Abelian gauge theory with $S U(3)$ gauge group. In this case, the gauge transformation is given by

$$
A_{\mu}^{\omega}(x)=\omega(x) A_{\mu}(x) \omega^{-1}(x)+\frac{i}{g} \omega(x) \partial_{\mu} \omega^{-1}(x)
$$

which gives in the infinitesimal form

$A_{\mu}^{a, \omega}(x)=A_{\mu}^{a}(x)+f^{a b c} A_{\mu}^{b}(x) \theta^{c}(x)+\frac{1}{g} \partial_{\mu} \theta^{a}(x)$,

where $\omega(x)=\exp \left(i \theta^{a}(x) t^{a}\right)$. The decomposition of field components in the longitudinal and transverse components is similar to the Abelian case, see above. In contrast to the $U(1)$ gauge group, the strength tensor $G_{\mu \nu}$ is gauge covariant. It means that all field components may change under gauge transformations.

\section{a. The Lorentz gauge}

We again begin with the Lorentz gauge condition:

$$
\begin{aligned}
\partial_{\mu} A_{\mu}^{a, \omega}(x) & =\partial_{\mu} A_{\mu}^{a}(x)+f^{a b c} \partial_{\mu}\left(A_{\mu}^{b}(x) \theta^{c}(x)\right)+\frac{1}{g} \partial^{2} \theta^{a}(x) \\
& =0
\end{aligned}
$$

As mentioned above, the gauge condition is invariant under the residual gauge transformation:

$$
\partial_{\mu} A_{\mu}^{a, \omega}(x)=\partial_{\mu} A_{\mu}^{a}(x)=0
$$

or, equivalently,

$$
\mathcal{D}_{\mu}^{a c} \partial_{\mu} \theta^{c}(x)=0,
$$

where $\mathcal{D}_{\mu}^{a c}=\partial_{\mu} \delta^{a c}+g f^{a b c} A_{\mu}^{b}(x)$.

In $p$-space, condition (B24) takes the form of

$$
-k^{2} \theta^{a}(k)+i g f^{a b c} k_{\mu} A_{\mu}^{b, L}(k) \theta^{c}(k)=0 .
$$

If $k^{2}=0$ and, therefore, $k_{\mu} A_{\mu}^{b, L}(k)=0$, then the gauge function $\theta(x)$ cannot be fixed and generates the residual gauge transformation.

\section{b. The Hamilton gauge}

A similar situation occurs in the Hamilton gauge, $A_{0}^{\omega}=0$. The residual transformation is induced by the gauge function which obeys

$$
\partial_{0} \theta^{a}\left(x_{0}, \vec{x}\right)=0
$$

Hence, the solution of this equation is rather trivial: $\theta$-function is the time-independent function, $\theta_{0}(\vec{x})$.

In the momentum representation, condition (B26) gives us the equation

$$
\int\left(d^{4} k\right) e^{i k x} k_{0} \theta^{a}\left(k_{0}, \vec{k}\right)=0
$$

which has a solution as

$$
\theta_{0}^{a}(k)=\delta\left(k_{0}\right) \theta_{0}^{a}(\vec{k}) .
$$

Therefore, we find in the coordinate representation

$$
\int\left(d^{4} k\right) e^{i k x} \delta\left(k_{0}\right) \theta_{0}^{a}(\vec{k})=\theta_{0}^{a}(\vec{x})
$$

which coincides with the results of the preceding paragraph.

\section{c. The axial gauge}

Working in the axial gauge, $A^{+, \omega}=0$, in a similar manner we are able to find the gauge function that is 
responsible for the residual gauge symmetry. We impose the condition

$$
A^{+, \omega}(x)=A^{+}(x)=0
$$

or, in the equivalent form,

$\partial^{+} \theta^{a}\left(x^{+}, x^{-}, \overrightarrow{\mathbf{x}}_{\perp}\right)=0 \quad$ with $\quad \partial^{+}=\partial_{-}=\frac{\partial}{\partial x^{-}}$.

The solution of this trivial differential equation is the $x^{-}$-independent function $\theta_{0}^{a}\left(x^{+}, \overrightarrow{\mathbf{x}}_{\perp}\right)$ which has the following form in $p$-space [cf. (B27) and (B28)]:

$$
\theta_{0}^{a}\left(k^{+}, k^{-}, \overrightarrow{\mathbf{k}}_{\perp}\right)=\delta\left(k^{+}\right) \theta_{0}^{a}\left(k^{-}, \overrightarrow{\mathbf{k}}_{\perp}\right),
$$

where $\theta_{0}^{a}\left(k^{-}, \overrightarrow{\mathbf{k}}_{\perp}\right)$ is an arbitrary gauge function related to the residual symmetry.

It is instructive to focus on the finite gauge transformations and corresponding gauge condition, namely

$$
\begin{aligned}
A^{+, \omega}(x) & =\omega(x) A^{+}(x) \omega^{-1}(x)+\frac{i}{g} \omega(x) \partial^{+} \omega^{-1}(x) \\
& =0 .
\end{aligned}
$$

The solution of this equation can easy be found; it reads

$$
\omega_{0}(x)=\mathbb{P} \exp \left\{i g \int_{\mathbb{C}}^{x^{-}} d z^{-} A^{+}\left(x^{+}, z^{-}, \overrightarrow{\mathbf{x}}_{\perp}\right)\right\},
$$

where, generally speaking, $\mathbb{C}$ is an arbitrary constant. We stress that the solution $\omega_{0}(x)$ is valid for $\forall x \in \mathbb{R}^{4}$. At the same time, this function can be multiplied by an arbitrary $x^{-}$-independent gauge function to produce another solution of Eq. (B33), i.e.

$$
W\left(x^{+}, x^{-}, \overrightarrow{\mathbf{x}}_{\perp}\right)=\bar{\omega}\left(x^{+}, \overrightarrow{\mathbf{x}}_{\perp}\right) \omega_{0}\left(x^{+}, x^{-}, \overrightarrow{\mathbf{x}}_{\perp}\right),
$$

where $\bar{\omega}\left(x^{+}, \overrightarrow{\mathbf{x}}_{\perp}\right)=\exp \left(i \theta^{a}\left(x^{+}, \overrightarrow{\mathbf{x}}_{\perp}\right) t^{a}\right)$. Indeed, one can demonstrate that $A^{+, W}(x)=0$.

To study the residual symmetry, we have to demand that $A^{+}(x)=0$ for any $x$. Therefore, from (B35), we obtain that the function

$$
\left.W\left(x^{+}, x^{-}, \overrightarrow{\mathbf{x}}_{\perp}\right)\right|_{A^{+}=0}=\bar{\omega}\left(x^{+}, \overrightarrow{\mathbf{x}}_{\perp}\right)
$$

generates the residual transformation we are interested in.

Let us now return to the gauge function presented by (B32). The case of $k^{+}=0$ (which provides us the residual symmetry) leads to the so-called spurious singularity in the gluon propagator in the axial gauge, see the next subsection. If we adopt a procedure to regularize this singularity with the help of some well-defined procedure, $\left[k^{+}\right]_{\text {reg }} \neq 0$, then the existence condition for the residual symmetry, see (B31), has to be given by (in the momentum representation)

$$
\int\left(d^{4} k\right) e^{i k x}\left[k^{+}\right]_{\mathrm{reg}} \delta\left(k^{+}\right) \theta_{0}^{a}\left(k^{-}, \overrightarrow{\mathbf{k}}_{\perp}\right)=0 .
$$

Hence, the only possibility to satisfy this equation is to demand that $\theta_{0}^{a}\left(k^{-}, \overrightarrow{\mathbf{k}}_{\perp}\right)=0$ which means that we fix the remaining residual symmetry. Thus, we conclude that the spurious singularity is fixed if and only if we do not have the residual gauge symmetry. On the other hand, we may say that the residual gauge fixing is enough for the elimination of spurious singularity.

\section{Spurious singularity of gluon propagator}

Let us return to the issue of the spurious singularity which appears in the gluon propagator in the axial gauge $A^{+}=0$.

The generating functional for gluons (gluonodynamics) in the most general gauge $F\left(A^{\theta}\right)=0$,

$$
\begin{aligned}
\mathbb{Z} & =N \int \mathcal{D} A_{\mu} e^{i S[A]} \\
& =\tilde{N} \int \mathcal{D} A_{\mu} \Delta_{c}[A] \delta(F(A)) e^{i S[A]},
\end{aligned}
$$

where $\tilde{N}$ involves the infinite gauge group volume, $\int d \theta$, and we use

$\mathbb{1}=\int d \theta \Delta_{c}[A] \delta\left(F\left(A^{\theta}\right)\right), \quad \Delta_{c}\left[A^{\theta}\right]=\Delta_{c}[A]$.

Instead of solving the gauge condition $F\left(A^{\theta}\right)=0$ with respect to the group function $\theta$ within the generalized Hamilton formalism, we separate out the infinite group volume, $\int d \theta$, in the generating functional (the FaddeevPopov approach).

The next trick is related to the exponentiation of $\delta(F(A))$. We introduce the generalized gauge condition as $F(A)=C$ with $\delta C / \delta A_{\mu}=0$. The generalizing functional $\mathbb{Z}$ must be independent on $C$. Therefore, to get the $C$-independent functional we have to integrate out over this parameter $C$. Using the integration measure defined as

$$
d C \exp \left(-\frac{i}{2 \xi} \int d^{4} x C^{2}(x)\right),
$$

we have

$$
\begin{aligned}
\mathbb{Z} & =\tilde{N} \int d C e^{\left(-\frac{i}{2 \xi} \int d^{4} x C^{2}(x)\right)} \int \mathcal{D} A_{\mu} \Delta_{c}[A] \delta(F(A)-C) e^{i S[A]} \\
& =\tilde{N} \int \mathcal{D} A_{\mu} \Delta_{c}[A] e^{i S[A]-\frac{i}{2 \xi} \int d^{4} x F^{2}(A)} .
\end{aligned}
$$


In (B41), the effective action with the gauge-fixing term,

$$
S_{\mathrm{fix}}=-\frac{1}{2 \xi} \int d^{4} x F^{2}(A),
$$

is now not gauge-invariant anymore. As a result of this trick, we do not need to solve the gauge condition with respect to the gauge function.

Let the gauge condition $F(A)=0$ be $A^{+}=0$ with $n^{2}=0$. In this case, the determinant $\Delta_{c}[A]$ is independent on $A$ and, therefore, we are able to include this determinant in the normalization of functional. Thus, the effective Lagrangian reads

$$
\mathcal{L}_{\text {eff }}=-\frac{1}{4} G_{\mu \nu} G_{\mu \nu}-\frac{1}{2 \xi}(n \cdot A)^{2} .
$$

This Lagrangian yields the effective action which can be written as

$$
S_{\mathrm{eff}}=\frac{1}{2} \int d^{4} x A_{\mu}(x) K_{\mu \nu}(x) A_{\nu}(x),
$$

where

$$
K_{\mu \nu}(x)=g_{\mu \nu} \partial^{2}-\partial_{\mu} \partial_{\nu}-\frac{1}{\xi} n_{\mu} n_{\nu} .
$$

In $p$-space, the operator $K_{\mu \nu}$ has an inverse operator which, in the limit of $\xi \rightarrow 0$, is given by

$$
\begin{aligned}
K_{\mu \nu}^{-1}(k) & =\frac{d_{\mu \nu}(k, n)}{k^{2}+i 0}, \\
d_{\mu \nu}(k, n) & =g_{\mu \nu}-\frac{k_{\mu} n_{\nu}+k_{\nu} n_{\mu}}{k^{+}} .
\end{aligned}
$$

As we have demonstrated in the preceding subsection, when we fix/regularize the spurious singularity $\left[k^{+}\right]_{\text {reg }}$ it means that we fix the residual gauge symmetry defined by the gauge function $\theta^{a}\left(k^{-}, \overrightarrow{\mathbf{k}}_{\perp}\right)$ and vice versa.

We also remind that it is not possible to fix the residual gauge simply by means of adding

$$
\frac{1}{2 \xi_{2}}\left(n^{*} \cdot A\right)^{2}
$$

in Eq. (B43). In this case, the inverse kinematical operator [see Eq. (B46)] does not exist due to the fact that the free (without the coefficients) tensors $n_{\mu} n_{\nu}$ and $n_{\mu}^{*} n_{\nu}^{*}$ present in the corresponding equation to determine the coefficients. Indeed, introducing the Lorentz parametrization (where the coefficients have to be determined)

$$
\begin{aligned}
d_{\nu \rho}\left(k, n, n^{*}\right)= & g_{\nu \rho}+a_{1} k_{\nu} k_{\rho}+b_{2} k_{\nu} n_{\rho}+b_{3} n_{\nu} k_{\rho}+b_{4} k_{\nu} n_{\rho}^{*} \\
& +b_{5} n_{\nu}^{*} k_{\rho}+c_{6} n_{\nu} n_{\rho}+c_{7} n_{\nu}^{*} n_{\rho}^{*}, \quad \text { (B48) }
\end{aligned}
$$

where

$$
\operatorname{dim}_{M}\left[a_{1}\right]=-2, \quad \operatorname{dim}_{M}\left[b_{i}\right]=-1, \quad \operatorname{dim}_{M}\left[c_{j}\right]=0,
$$

the contraction equation on the coefficients (or, in other words, the Green function equation)

$$
K_{\mu \nu} d_{\nu \rho}=g_{\mu \rho}
$$

involves the tensors $n_{\mu} n_{\nu}$ and $n_{\mu}^{*} n_{\nu}^{*}$ which stay without coefficients. It means that the inverse operator cannot be derived.
[1] R. Angeles-Martinez et al., Transverse momentum dependent (TMD) parton distribution functions: Status and prospects, Acta Phys. Pol. B 46, 2501 (2015).

[2] D. Boer et al., Gluons and the quark sea at high energies: Distributions, polarization, tomography, arXiv:1108.1713.

[3] D. Boer, P. J. Mulders, and F. Pijlman, Universality of T odd effects in single spin and azimuthal asymmetries, Nucl. Phys. B667, 201 (2003).

[4] Z. B. Kang, J. W. Qiu, W. Vogelsang, and F. Yuan, An observation concerning the process dependence of the Sivers functions, Phys. Rev. D 83, 094001 (2011).

[5] D. Boer, On a possible node in the Sivers and Qiu-Sterman functions, Phys. Lett. B 702, 242 (2011).
[6] B. Pire and J. P. Ralston, Single spin asymmetries in the Drell-Yan process, Phys. Rev. D 28, 260 (1983).

[7] R. D. Carlitz and R. S. Willey, Single spin asymmetries in muon pair production, Phys. Rev. D 45, 2323 (1992).

[8] A. Brandenburg, D. Mueller, and O. V. Teryaev, Extraction of the pion distribution amplitude from polarized muon pair production, Phys. Rev. D 53, 6180 (1996).

[9] A. P. Bakulev, N. G. Stefanis, and O. V. Teryaev, Polarized and unpolarized $\mu$-pair meson-induced Drell-Yan production and the pion distribution amplitude, Phys. Rev. D 76, 074032 (2007).

[10] A. V. Radyushkin, Shape of pion distribution amplitude, Phys. Rev. D 80, 094009 (2009). 
[11] M. V. Polyakov, On the pion distribution amplitude shape, JETP Lett. 90, 228 (2009).

[12] S. V. Mikhailov and N. G. Stefanis, Pion transition form factor at the two-loop level vis-à-vis experimental data, Mod. Phys. Lett. A 24, 2858 (2009).

[13] N. Hammon, O. Teryaev, and A. Schafer, Single spin asymmetry for the Drell-Yan process, Phys. Lett. B 390, 409 (1997).

[14] D. Boer, P. J. Mulders, and O. V. Teryaev, Single spin asymmetries from a gluonic background in the Drell-Yan process, Phys. Rev. D 57, 3057 (1998).

[15] I. V. Anikin and O. V. Teryaev, New contributions to gluon poles in direct photon production, Eur. Phys. J. C 75, 184 (2015); Constraining gluon poles, Phys. Lett. B 751, 495 (2015); Gauge invariance, causality and gluonic poles, Phys. Lett. B 690, 519 (2010).

[16] A. V. Belitsky, X. Ji, and F. Yuan, Final state interactions and gauge invariant parton distributions, Nucl. Phys. B656, 165 (2003).

[17] G. A. Chirilli, Y. V. Kovchegov, and D. E. Wertepny, Regularization of the light-cone gauge gluon propagator singularities using sub-gauge conditions, J. High Energy Phys. 12 (2015) 138.

[18] D. E. Soper, Partons and Their Transverse Momenta in QCD, Phys. Rev. Lett. 43, 1847 (1979).

[19] J. C. Collins and D. E. Soper, Parton distribution and decay functions, Nucl. Phys. B194, 445 (1982).

[20] G. Leibbrandt, Introduction to noncovariant gauges, Rev. Mod. Phys. 59, 1067 (1987).

[21] A. A. Slavnov and S. A. Frolov, Propagator of Yang-Mills field in light cone gauge, Theor. Math. Phys. 73, 1158 (1987) [Teor. Mat. Fiz. 73, 199 (1987)].

[22] A. Bassetto, G. Nardelli, and R. Soldati, Yang-Mills Theories in Algebraic Noncovariant Gauges: Canonical Quantization and Renormalization (World Scientific, Singapore, 1991), p. 227.

[23] A. Bassetto, M. Dalbosco, I. Lazzizzera, and R. Soldati, Yang-Mills theories in the light cone gauge, Phys. Rev. D 31, 2012 (1985).

[24] J. C. Collins, D. E. Soper, and G. F. Sterman, Factorization of hard processes in QCD, Adv. Ser. Dir. High Energy Phys. 5, 1 (1989).

[25] S. V. Ivanov, Contour gauges in non-Abelian gauge theories, Fiz. Elem. Chastits At. Yadra 21, 75 (1990).
[26] N. G. Stefanis, Gauge invariant quark two point Green's function through connector insertion to O (alpha-s), Nuovo Cimento A 83, 205 (1984).

[27] V. Barone, A. Drago, and P. G. Ratcliffe, Transverse polarisation of quarks in hadrons, Phys. Rep. 359, 1 (2002).

[28] A. V. Efremov and O. V. Teryaev, QCD asymmetry and polarized hadron structure functions, Phys. Lett. B 150, 383 (1985).

[29] I. V. Anikin, D. Y. Ivanov, B. Pire, L. Szymanowski, and S. Wallon, QCD factorization of exclusive processes beyond leading twist: $\gamma * T \rightarrow \rho(T)$ impact factor with twist three accuracy, Nucl. Phys. B828, 1 (2010).

[30] I. V. Anikin and O. V. Teryaev, Factorization and transverse momentum in double inclusive $e+e-$ annihilation, Phys. Part. Nucl. Lett. 6, 3 (2009).

[31] V. M. Braun, D. Y. Ivanov, A. Schafer, and L. Szymanowski, Towards the theory of coherent hard dijet production on hadrons and nuclei, Nucl. Phys. B638, 111 (2002).

[32] N. N. Bogolyubov and D. V. Shirkov, Introduction to the theory of quantized fields, Interscience monographs and texts in Physics and astronomy 3, 1 (1959).

[33] A. V. Efremov and A. V. Radyushkin, Field theoretic treatment of high momentum transfer processes. 3. Gauge theories, Theor. Math. Phys. 44, 774 (1981) [Teor. Mat. Fiz.44, 327 (1980)].

[34] S. V. Ivanov, G. P. Korchemsky, and A. V. Radyushkin, Infrared asymptotics of perturbative QCD: Contour gauges, Yad. Fiz. 44, 230 (1986) [Sov. J. Nucl. Phys. 44, 145 (1986)]; S. V. Ivanov and G. P. Korchemsky, Some supplements of nonperturbative gauges, Phys. Lett. B 154, 197 (1985).

[35] V. S. Vladimirov, Generalized Functions in Mathematical Physics (Mir Publishers, Moscow, 1979), p. 390.

[36] D. J. Gross and S. B. Treiman, Light cone structure of current commutators in the gluon quark model, Phys. Rev. D 4, 1059 (1971).

[37] I. I. Balitsky and V. M. Braun, Evolution equations for QCD string operators, Nucl. Phys. B311, 541 (1989).

[38] A. V. Radyushkin, Analysis of the hard inclusive processes in quantum chromodynamics, Fiz. Elem. Chastits At. Yadra 14, 58 (1983).

[39] V. A. Rubakov, Classical Theory of Gauge Fields (University Press, Princeton, NJ, 2002), p. 444. 